\title{
Gluon polarization tensor in a magnetized medium: Analytic approach starting from the sum over Landau levels
}

\author{
Alejandro Ayala@ ${ }^{1,2}$ Jorge David Castaño-Yepes $\odot,{ }^{1, *}$ M. Loewe, ${ }^{3,2,4}$ and Enrique Muñoz $\odot^{3,5}$ \\ ${ }^{1}$ Instituto de Ciencias Nucleares, Universidad Nacional Autónoma de México, \\ Apartado Postal 70-543, CdMx 04510, Mexico \\ ${ }^{2}$ Centre for Theoretical and Mathematical Physics, and Department of Physics, \\ University of Cape Town, Rondebosch 7700, South Africa \\ ${ }^{3}$ Instituto de Física, Pontificia Universidad Católica de Chile, Casilla 306, Santiago 22, Chile \\ ${ }^{4}$ Centro Científico-Tecnológico de Valparaíso CCTVAL, Universidad Técnica Federico Santa María, \\ Casilla 110-V, Valparaíso, Chile \\ ${ }^{5}$ Research Center for Nanotechnology and Advanced Materials CIEN-UC, \\ Pontificia Universidad Catolica de Chile, Casilla 306, Santiago 22, Chile
}

(Received 17 December 2019; accepted 28 January 2020; published 18 February 2020)

\begin{abstract}
We present an analytic method to compute the one-loop magnetic correction to the gluon polarization tensor starting from the Landau-level representation of the quark propagator in the presence of an external magnetic field. We show that the general expression contains the vacuum contribution that can be isolated from the zero-field limit for finite gluon momentum. The general tensor structure for the gluon polarization also contains two spurious terms that do not satisfy the transversality properties. However, we also show that the coefficients of these structures vanish and thus do not contribute to the polarization tensor, as expected. In order to check the validity of the expressions we study the strong and weak field limits and show that in the former, the well-established result is reproduced. The findings can be used to study the conditions for gluons to equilibrate with the magnetic field produced during the early stages of a relativistic heavy-ion collision.
\end{abstract}

DOI: 10.1103/PhysRevD.101.036016

\section{INTRODUCTION}

The production of hot and dense strongly interacting matter in heavy-ion reactions at high energies constitutes a driving force for the formulation of novel approaches to study QCD subject to extreme conditions. For semicentral collisions, these conditions include the presence of strong, albeit short-lived, magnetic fields. Many theoretical efforts concentrate on describing these conditions considering that the temperature is the largest of the energy scales [1-4]. However, it has also been realized that the imprints of these strong fields [5,6], if any, should be searched for studying probes produced during the very early stages of the collision, where the system is not yet equilibrated and the largest of the energy scales is instead the magnetic field itself. Possible imprints include an enhanced prompt photon production and/or the chiral magnetic effect [7-12].

\footnotetext{
*jorgecastanoy@gmail.com
}

Published by the American Physical Society under the terms of the Creative Commons Attribution 4.0 International license. Further distribution of this work must maintain attribution to the author(s) and the published article's title, journal citation, and DOI. Funded by SCOAP.
The early stages of a heavy-ion reaction are also characterized by the presence of a large number of low momentum gluons which are thought to give rise to the saturation phenomenon described by the Glasma [13]. When a magnetic field is present, gluon dynamics can also be affected. A deeper understanding of gluon properties within a magnetized medium is crucial for describing the evolution of observables coming from these early stages.

The gluon dispersive properties in a magnetized medium are encoded in the gluon polarization tensor $\Pi^{\mu \nu}$. In a perturbative approach, deviations from its vacuum properties come from the coupling of the magnetic field to virtual quarks. The quark propagator can be represented in terms of a sum over Landau levels. When the field is strong, calculations often resort to the approximation where these quarks occupy the lowest Landau level (LLL), which simplifies considerably the treatment [14-16]. Nevertheless, when the field is not as intense, it is important to perform a sum over Landau levels to capture effects that may be missing from expressions restricted to the LLL, in particular, the emergence of tensor polarization structures other than the parallel one that makes up the full polarization tensor. These kinds of calculations have been performed at one-loop level for the photon polarization tensor [17] in the context of the vacuum 
birefringence in strong magnetic fields, where the authors resort to a numerical treatment for the infinite sum over Landau levels. However, in order to gain a deeper insight, an analytical approach for the infinite sum over Landau levels is desirable.

In this work, we undertake such task and present an analytic method to perform the sum over all Landau levels for the coefficients of the tensor structure that make up the gluon polarization tensor in the presence of a magnetic field of arbitrary intensity. The vacuum contribution is obtained in the limit when $B \rightarrow 0$. We show that by this procedure one obtains the usual fermion contribution to the vacuum polarization tensor, together with a second term that is shown to vanish, given the properties of its coefficient under scaling transformations. Applying the same argument to the full, magnetic field-dependent polarization tensor, it is possible to isolate the physical tensor structures and their coefficients, thus getting rid of spurious terms. We then proceed to carefully subtract the vacuum pieces to remove ultraviolet divergences. The procedure ensures that the remaining, magnetic field-dependent contributions are finite. In order to test the validity of the expressions thus obtained, we study the weak and strong magnetic field limits. The work is organized as follows: In Sec. II, we write the one-loop expression for the gluon polarization tensor in the presence of a constant external magnetic field. We chose the tensor basis to express the polarization tensor and outline the calculation to carry out the product of fermion propagators and the corresponding sums over Landau levels. We show that after the sum is made, there appear two spurious, nontransverse terms. These are shown to vanish, as in the vacuum case, from the properties of their coefficients under scaling transformations. In Sec. III we study the strong and in Sec. IV the weak field limits and show that the obtained expressions coincide with well-known results. We summarize and discuss our results in Sec. V and leave for the appendixes the calculation details.

\section{GLUON POLARIZATION TENSOR}

We start from the one-loop contribution to the gluon polarization tensor, which is depicted in Fig. 1 and is given explicitly by

$$
\begin{aligned}
i \Pi_{a b}^{\mu \nu}= & -\frac{1}{2} \int \frac{d^{4} k}{(2 \pi)^{4}} \operatorname{Tr}\left\{i g t_{b} \gamma^{\nu} i S^{(n)}(k) i g t_{a} \gamma^{\mu} i S^{(m)}(q)\right\} \\
& + \text { C.C. },
\end{aligned}
$$

where C.C. refers to the charge conjugate contribution, that is, the contribution where the flow of charge within the loop is in the opposite direction. The factor $1 / 2$ accounts for the symmetry factor, which in the presence of the external magnetic field comes about given that the two contributing diagrams, with the opposite flow of charge, are not equivalent. Also $g$ is the strong coupling. $S(k)$ is the quark propagator and $t_{a, b}$ are the generators of the color group in

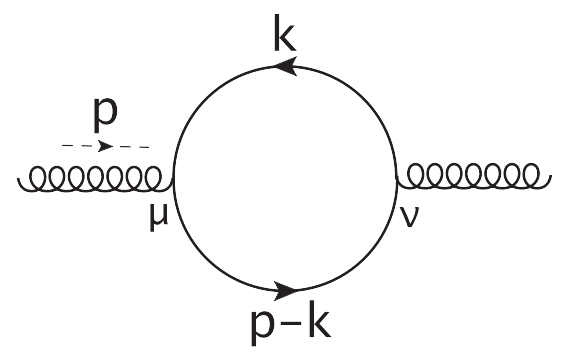

FIG. 1. One-loop diagram representing the gluon polarization tensor.

the fundamental representation. The fermion propagator in the presence of a magnetic field $\vec{B}=B \hat{z}$ can be written in terms of a sum over Landau levels as $[18,19]$

$$
i S(p)=i e^{-p_{\perp}^{2} /\left|q_{f} B\right|} \sum_{n=0}^{+\infty}(-1)^{n} \frac{D_{n}\left(q_{f} B, p\right)}{p_{\|}^{2}-m_{f}^{2}-2 n\left|q_{f} B\right|},
$$

where $m_{f}$ and $q_{f}$ are the quark mass and electric charge, respectively, and

$$
\begin{aligned}
D_{n}\left(q_{f} B, p\right)= & 2\left(\not \|_{\|}+m_{f}\right) \mathcal{O}^{-} L_{n}^{0}\left(\frac{2 p_{\perp}^{2}}{\left|q_{f} B\right|}\right) \\
& -2\left(\not \not_{\|}+m_{f}\right) \mathcal{O}^{+} L_{n-1}^{0}\left(\frac{2 p_{\perp}^{2}}{\left|q_{f} B\right|}\right) \\
& +4 \not \not_{\perp} L_{n-1}^{1}\left(\frac{2 p_{\perp}^{2}}{\left|q_{f} B\right|}\right) .
\end{aligned}
$$

In Eq. (3), $L_{n}^{\alpha}(x)$ are the generalized Laguerre polynomials, with the index $n$ labeling the $n$th Landau level, and

$$
\mathcal{O}^{( \pm)}=\frac{1}{2}\left[1 \pm i \gamma^{1} \gamma^{2} \operatorname{sign}\left(q_{f} B\right)\right]
$$

Also, we follow the convention whereby the square of the four-momentum $p^{\mu}$, expressed in terms of the square of its parallel and perpendicular (with respect to the magnetic field direction) components, is given by

$$
p^{2}=p_{\|}^{2}-p_{\perp}^{2}=\left(p_{0}^{2}-p_{3}^{2}\right)-\left(p_{1}^{2}+p_{2}^{2}\right) .
$$

Computing Eqs. (1) and (2), after performing the sum over all Landau levels, the gluon polarization tensor can be written in terms of four tensor structures, given by

$$
i \Pi^{\mu \nu}=-\frac{i}{8 \pi^{2}} g^{2} \int d^{2} x f_{0}\left(x_{1}, x_{2}\right) \sum_{i=1}^{4} f_{i}^{\mu \nu}\left(x_{1}, x_{2}\right),
$$

where on the right-hand side, we have omitted a factor $\delta_{a b}$ coming from using the relation $\operatorname{Tr}\left(t^{a} t^{b}\right)=\delta_{a b} / 2$, and correspondingly, for notation simplicity, removed the color indices on the left-hand side. Here $\left(x_{1}, x_{2}\right) \in(0, \infty)$ are Schwinger parameters, with $d^{2} x=d x_{1} d x_{2}$ and 


$$
\begin{gathered}
f_{0}\left(x_{1}, x_{2}\right)=\exp \left[\frac{x_{1} x_{2}}{x_{1}+x_{2}} p_{\|}^{2}-m_{f}^{2}\left(x_{1}+x_{2}\right)\right] \exp \left[-\frac{\tanh \left(\left|q_{f} B\right| x_{1}\right) \tanh \left(\left|q_{f} B\right| x_{2}\right)}{\tanh \left(\left|q_{f} B\right| x_{1}\right)+\tanh \left(\left|q_{f} B\right| x_{2}\right)\left|q_{f} B\right|}\right], \\
f_{1}^{\mu \nu}\left(x_{1}, x_{2}\right)=\left|q_{f} B\right| \operatorname{coth}\left[\left|q_{f} B\right|\left(x_{1}+x_{2}\right)\right]\left[\left(\frac{x_{1} x_{2}}{\left(x_{1}+x_{2}\right)^{3}} p_{\|}^{2}+\frac{m_{f}^{2}}{x_{1}+x_{2}}\right) g_{\|}^{\mu \nu}-\frac{2 x_{1} x_{2}}{\left(x_{1}+x_{2}\right)^{3}} p_{\|}^{\mu} p_{\|}^{\nu}\right], \\
f_{2}^{\mu \nu}\left(x_{1}, x_{2}\right)=\left|q_{f} B\right| \frac{\cosh \left[\left|q_{f} B\right|\left(x_{2}-x_{1}\right)\right]}{\sinh \left[\left|q_{f} B\right|\left(x_{1}+x_{2}\right)\right]}\left[\frac{x_{1} x_{2}}{\left(x_{1}+x_{2}\right)^{3}} p_{\|}^{2}+\frac{m_{f}^{2}}{x_{1}+x_{2}}+\frac{1}{\left(x_{1}+x_{2}\right)^{2}}\right] g_{\perp}^{\mu \nu}, \\
f_{3}^{\mu \nu}\left(x_{1}, x_{2}\right)=\frac{\left|q_{f} B\right|}{2\left(x_{1}+x_{2}\right)^{2} \sinh ^{2}\left[\left|q_{f} B\right|\left(x_{1}+x_{2}\right)\right]}\left[x_{1} \sinh \left(2\left|q_{f} B\right| x_{2}\right)+x_{2} \sinh \left(2\left|q_{f} B\right| x_{1}\right)\right]\left(p_{\|}^{\mu} p_{\perp}^{\nu}+p_{\|}^{\nu} p_{\perp}^{\mu}\right), \\
f_{4}^{\mu \nu}\left(x_{1}, x_{2}\right)=\frac{\left|q_{f} B\right|^{2}}{\left(x_{1}+x_{2}\right) \sinh ^{2}\left[\left|q_{f} B\right|\left(x_{1}+x_{2}\right)\right]}\left[\left(1-\frac{\tanh \left(\left|q_{f} B\right| x_{1}\right) \tanh \left(\left|q_{f} B\right| x_{2}\right)}{\left|q_{f} B\right|\left[\tanh \left(\left|q_{f} B\right| x_{1}\right)+\tanh \left(\left|q_{f} B\right| x_{2}\right)\right]} p_{\perp}^{2}\right) g^{\mu \nu}\right. \\
\left.-g_{\perp}^{\mu \nu}-\frac{2 \tanh \left(\left|q_{f} B\right| x_{1}\right) \tanh \left(\left|q_{f} B\right| x_{2}\right)}{\left|q_{f} B\right|\left[\tanh \left(\left|q_{f} B\right| x_{1}\right)+\tanh \left(\left|q_{f} B\right| x_{2}\right)\right]} p_{\perp}^{\mu} p_{\perp}^{\nu}\right] .
\end{gathered}
$$

For calculation details, see Appendix A.

\section{A. Tensor basis}

The gluon polarization tensor should be represented by a symmetric tensor under the exchange of its Lorentz indices. It can be constructed out of the external products of the independent vectors describing the propagation of a gluon with momentum $p^{\mu}$ in the presence of a magnetic field whose direction is specified by a four-vector $b^{\mu}$, in addition to the metric tensor $g^{\mu \nu}$. Without loss of generality, we can choose a reference frame where the magnetic field points along the $\hat{z}$ axis. Due to the presence of this Lorentz invariance-breaking vector, it is convenient to split the metric itself into parallel and perpendicular (with respect to the magnetic field direction) components, that is

$$
g^{\mu \nu}=g_{\|}^{\mu \nu}+g_{\perp}^{\mu \nu}
$$

where

$$
g_{\|}^{\mu \nu}=\operatorname{diag}(1,0,0,-1),
$$

and

$$
g_{\perp}^{\mu \nu}=\operatorname{diag}(0,-1,-1,0) .
$$

We thus see that the most general symmetric tensor can be constructed out of combinations of the four possible independent tensors

$$
p^{\mu} p^{\nu}, \quad b^{\mu} b^{\nu}, \quad p^{\mu} b^{\nu}+p^{\nu} b^{\mu}, \quad g^{\mu \nu} .
$$

However, notice that in QCD, $\Pi^{\mu \nu}$ must satisfy the generalized Ward-Takahashi identity namely, the transversality condition

$$
p_{\mu} p_{\nu} \Pi^{\mu \nu}=0 .
$$

Therefore, since Eq. (12) implies a relation between the coefficients of the tensors to express $\Pi^{\mu \nu}$, only three transverse tensors turn out to be independent. To visualize this, let us suppose that $\Pi^{\mu \nu}$ can be written as

$$
\Pi^{\mu \nu}=a A^{\mu \nu}+b B^{\mu \nu}+c C^{\mu \nu}+d D^{\mu \nu} .
$$

Gauge invariance, Eq. (12), implies

$$
\begin{aligned}
p_{\mu} p_{\nu} \Pi^{\mu \nu}= & a\left(p_{\mu} p_{\nu} A^{\mu \nu}\right)+b\left(p_{\mu} p_{\nu} B^{\mu \nu}\right) \\
& +c\left(p_{\mu} p_{\nu} C^{\mu \nu}\right)+d\left(p_{\mu} p_{\nu} D^{\mu \nu}\right)=0 .
\end{aligned}
$$

Equation (14) means that only three out of the four factors $(a, b, c, d)$ are independent. Therefore, the tensor structure that multiplies the factor chosen as not independent can be distributed among the rest of the structures to result in only three of them being needed to span the whole tensor $\Pi^{\mu \nu}$. A convenient basis to express the polarization tensor is such that the independent tensors are chosen each to be transverse, in such a way that Eq. (12) be satisfied already as

$$
p_{\mu} \Pi^{\mu \nu}=0 .
$$

This choice has the advantage that the basis can be used to express the polarization tensor either in QCD or in QED. In the present work, we chose the orthonormal basis

$$
\begin{gathered}
\mathcal{P}_{\|}^{\mu \nu}=g_{\|}^{\mu \nu}-\frac{p_{\|}^{\mu} p_{\|}^{\nu}}{p_{\|}^{2}}, \\
\mathcal{P}_{\perp}^{\mu \nu}=g_{\perp}^{\mu \nu}+\frac{p_{\perp}^{\mu} p_{\perp}^{\nu}}{p_{\perp}^{2}},
\end{gathered}
$$




$$
\mathcal{P}_{0}^{\mu \nu}=g^{\mu \nu}-\frac{p^{\mu} p^{\nu}}{p^{2}}-\mathcal{P}_{\|}^{\mu \nu}-\mathcal{P}_{\perp}^{\mu \nu}
$$

Such choice comes from the factorization of the metric into transverse and parallel structures induced by the presence of the vector $b^{\mu}$ representing the direction of the magnetic field. To show this, we can choose $b^{\mu}=(0, \mathbf{b})=(0,0,0,1)$. Introducing the space-vector $\mathbf{a}=(1 / 2)(-y, x, 0)$ such that $\mathbf{b}=\nabla \times \mathbf{a}$, we observe that by choosing the vector potential as $\mathbf{A}=B \mathbf{a}$, and from the definition $F^{\mu \nu}=\partial^{\mu} A^{\nu}-$ $\partial^{\nu} A^{\mu}$ we get

$$
p_{\alpha} p_{\beta} F^{\alpha \mu} F^{\beta \nu}=B^{2}\left(\begin{array}{cccc}
0 & 0 & 0 & 0 \\
0 & p_{2}^{2} & -p_{1} p_{2} & 0 \\
0 & -p_{1} p_{2} & p_{1}^{2} & 0 \\
0 & 0 & 0 & 0
\end{array}\right) .
$$

Also, from Eq. (17)

$$
p_{\perp}^{2} \mathcal{P}_{\perp}^{\mu \nu}=\left(\begin{array}{cccc}
0 & 0 & 0 & 0 \\
0 & -p_{2}^{2} & p_{1} p_{2} & 0 \\
0 & p_{1} p_{2} & -p_{1}^{2} & 0 \\
0 & 0 & 0 & 0
\end{array}\right),
$$

therefore

$$
B^{2} p_{\perp}^{2} \mathcal{P}_{\perp}^{\mu \nu}=-p_{\alpha} p_{\beta} F^{\alpha \mu} F^{\beta \nu},
$$

which shows that the choice of $b^{\mu}$ impacts directly the factorization of the metric into transverse and parallel structures.

On the other hand, notice that when Eqs. (16)-(18) are chosen as the basis to span $\Pi^{\mu \nu}$, the condition of Eq. (15) does not reduce the number of independent tensor structures from three to two, given that the tensor structures are already transverse.

Therefore, we can use this basis (see also Ref. [20]) to express Eqs. (7) (see Appendix B) as

$$
i \Pi^{\mu \nu}=-\frac{i}{8 \pi^{2}} g^{2} \int d^{2} x f_{0}\left(x_{1}, x_{2}\right)\left[\Pi_{\|}\left(x_{1}, x_{2}\right) \mathcal{P}_{\|}^{\mu \nu}+\Pi_{\perp}\left(x_{1}, x_{2}\right) \mathcal{P}_{\perp}^{\mu \nu}+\Pi_{0}\left(x_{1}, x_{2}\right) \mathcal{P}_{0}^{\mu \nu}+A_{1}\left(x_{1}, x_{2}\right) g_{\|}^{\mu \nu}+A_{2}\left(x_{1}, x_{2}\right) g_{\perp}^{\mu \nu}\right],
$$

where

$$
\begin{gathered}
\Pi_{\|}=\left|q_{f} B\right|\left[\frac{2 x_{1} x_{2} \operatorname{coth}\left[\left|q_{f} B\right|\left(x_{1}+x_{2}\right)\right]}{\left(x_{1}+x_{2}\right)^{3}} p_{\|}^{2}-\frac{x_{1} \sinh \left(2\left|q_{f} B\right| x_{2}\right)+x_{2} \sinh \left(2\left|q_{f} B\right| x_{1}\right)}{2\left(x_{1}+x_{2}\right)^{2} \sinh ^{2}\left[\left|q_{f} B\right|\left(x_{1}+x_{2}\right)\right]} p_{\perp}^{2}\right], \\
\Pi_{\perp}=\left|q_{f} B\right|\left[\frac{x_{1} \sinh \left(2\left|q_{f} B\right| x_{2}\right)+x_{2} \sinh \left(2\left|q_{f} B\right| x_{1}\right)}{2\left(x_{1}+x_{2}\right)^{2} \sinh ^{2}\left[\left|q_{f} B\right|\left(x_{1}+x_{2}\right)\right]} p_{\|}^{2}-\frac{2 \sinh \left(\left|q_{f} B\right| x_{1}\right) \sinh \left(\left|q_{f} B\right| x_{2}\right)}{\left(x_{1}+x_{2}\right) \sinh ^{3}\left[\left|q_{f} B\right|\left(x_{1}+x_{2}\right)\right]} p_{\perp}^{2}\right], \\
\Pi_{0}=\left|q_{f} B\right| \frac{x_{1} \sinh \left(2\left|q_{f} B\right| x_{2}\right)+x_{2} \sinh \left(2\left|q_{f} B\right| x_{1}\right)}{2\left(x_{1}+x_{2}\right)^{2} \sinh ^{2}\left[\left|q_{f} B\right|\left(x_{1}+x_{2}\right)\right]} p^{2}, \\
A_{1}=\left|q_{f} B\right|\left[\frac{x_{1} \sinh \left(2\left|q_{f} B\right| x_{2}\right)+x_{2} \sinh \left(2\left|q_{f} B\right| x_{1}\right)}{2\left(x_{1}+x_{2}\right)^{2} \sinh ^{2}\left[\left|q_{f} B\right|\left(x_{1}+x_{2}\right)\right]} p_{\perp}^{2}+\frac{\operatorname{coth}\left[\left|q_{f} B\right|\left(x_{1}+x_{2}\right)\right]}{\left(x_{1}+x_{2}\right)^{3}}\left(m_{f}^{2}\left(x_{1}+x_{2}\right)^{2}-x_{1} x_{2} p_{\|}^{2}\right)\right. \\
\left.+\frac{\left|q_{f} B\right|}{\left(x_{1}+x_{2}\right) \sinh ^{2}\left[\left|q_{f} B\right|\left(x_{1}+x_{2}\right)\right]} \times\left(1-\frac{\tanh \left(\left|q_{f} B\right| x_{1}\right) \tanh \left(\left|q_{f} B\right| x_{2}\right)}{\left|q_{f} B\right|\left[\tanh \left(\left|q_{f} B\right| x_{1}\right)+\tanh \left(\left|q_{f} B\right| x_{2}\right)\right]} p_{\perp}^{2}\right)\right],
\end{gathered}
$$

and

$$
\begin{aligned}
A_{2}= & \left|q_{f} B\right|\left[\frac{\cosh \left[\left|q_{f} B\right|\left(x_{2}-x_{1}\right)\right]}{\left(x_{1}+x_{2}\right)^{3} \sinh \left[\left|q_{f} B\right|\left(x_{1}+x_{2}\right)\right]}\left[x_{1} x_{2} p_{\|}^{2}+\left(x_{1}+x_{2}\right)+m_{f}^{2}\left(x_{1}+x_{2}\right)^{2}\right]\right. \\
& \left.-\frac{x_{1} \sinh \left(2\left|q_{f} B\right| x_{2}\right)+x_{2} \sinh \left(2\left|q_{f} B\right| x_{1}\right)}{2\left(x_{1}+x_{2}\right)^{2} \sinh ^{2}\left[\left|q_{f} B\right|\left(x_{1}+x_{2}\right)\right]} p_{\|}^{2}+\frac{\sinh \left(\left|q_{f} B\right| x_{1}\right) \sinh \left(\left|q_{f} B\right| x_{2}\right)}{\left(x_{1}+x_{2}\right) \sinh ^{3}\left[\left|q_{f} B\right|\left(x_{1}+x_{2}\right)\right]} p_{\perp}^{2}\right] .
\end{aligned}
$$

Notice that, contrary to expectations, Eq. (22) contains also terms proportional to the tensors $g_{\|}^{\mu \nu}$ and $g_{\perp}^{\mu \nu}$. In order to show that $\Pi^{\mu \nu}$ is made out only of combinations of transverse tensors, we need to prove that the coefficients
$A_{1}$ and $A_{2}$ vanish. This is shown in Appendix C. For the time being, let us only emphasize that, had we simply projected out Eq. (6) onto the basis given by Eqs. (23)-(25), the spurious terms would have induced nonphysical 
contributions that, given their complexity, could obscure the numerical evaluation of the physical coefficients $[17,21,23]$. This comes about since, formally, a simple projection would give rise to the tensor coefficients

$$
\begin{gathered}
\tilde{\Pi}_{\|}=\Pi_{\mu \nu} \mathcal{P}_{\|}^{\mu \nu}=\Pi_{\|}+A_{1}, \\
\tilde{\Pi}_{\perp}=\Pi_{\mu \nu} \mathcal{P}_{\perp}^{\mu \nu}=\Pi_{\perp}+A_{2},
\end{gathered}
$$

and

$$
\tilde{\Pi}_{0}=\Pi_{\mu \nu} \mathcal{P}_{0}^{\mu \nu}=\Pi_{0}-\frac{p_{\perp}^{2}}{p^{2}} A_{1}+\frac{p_{\|}^{2}}{p^{2}} A_{2},
$$

where $\Pi_{\|}, \Pi_{\perp}$ and $\Pi_{0}$ are given by Eqs. (23)-(25) and $A_{1}, A_{2}$ are given by Eqs. (26) and (27), showing that such projection contains spurious terms.

\section{B. Vacuum polarization tensor}

As one can expect, the gluon polarization tensor contains divergences which come from the vacuum contribution. In order to proceed to isolate these contributions we notice that two possible vacua can be defined:

(1) a vacuum where $p^{\mu}=0$ and $B=0$, corresponding to a situation where particles and magnetic field appear simultaneously, and

(2) a vacuum with $B=0$ and $p^{\mu} \neq 0$, representing a situation where the external field is turned on with preexisting gluons with four-momentum $p^{\mu}$.

The first choice is ambiguous, given that the energy scales associated with the magnetic field and the transverse momentum appear within the combination $p_{\perp}^{2} /\left|q_{f} B\right|$, and thus, $p_{\perp}^{2}$ and $B$ cannot be set to zero simultaneously. Therefore, we chose to extract the vacuum working in the situation described by the second case. The vacuum contribution is thus given by

$$
\begin{aligned}
i \Pi^{\mu \nu}\left(p,\left|q_{f} B\right| \rightarrow 0\right) \\
=-\frac{i}{8 \pi^{2}} g^{2} \int d^{2} x \exp \left[\frac{x_{1} x_{2}}{x_{1}+x_{2}} p^{2}-m_{f}^{2}\left(x_{1}+x_{2}\right)\right] \\
\quad \times\left[\frac{2 x_{1} x_{2}}{\left(x_{1}+x_{2}\right)^{4}} p^{2}\left(g^{\mu \nu}-\frac{p^{\mu} p^{\nu}}{p^{2}}\right)\right. \\
\left.\quad+\frac{1}{\left(x_{1}+x_{2}\right)^{3}}\left(\left(x_{1}+x_{2}\right) m_{f}^{2}-\frac{x_{1} x_{2}}{x_{1}+x_{2}} p^{2}+1\right) g^{\mu \nu}\right] .
\end{aligned}
$$

Notice that Eq. (29) contains a term that does not simply vanish under contraction with $p_{\mu}$, namely, the term proportional to $g^{\mu \nu}$. In order to show that the coefficient of this term vanishes, we follow the argument in Ref. [22]. We introduce the scaling transformation for the Schwinger parameters in such a way that $x_{i} \rightarrow \lambda z_{i}$, where $\lambda$ is a real parameter. Under this transformation, the coefficient of the term proportional to $g^{\mu \nu}$ becomes

$$
\begin{aligned}
\mathcal{I}= & \lambda^{2} \int \frac{d^{2} z}{\lambda^{2}\left(z_{1}+z_{2}\right)^{3}}\left(m^{2}\left(z_{1}+z_{2}\right)-\frac{z_{1} z_{2}}{z_{1}+z_{2}} p^{2}+\frac{1}{\lambda}\right) \\
& \times \exp \left[\lambda\left(\frac{z_{1} z_{2}}{z_{1}+z_{2}} p^{2}-m_{f}^{2}\left(z_{1}+z_{2}\right)\right)\right] .
\end{aligned}
$$

It is easy to show that the integral $\mathcal{I}$ can also be written as

$$
\mathcal{I}=-\lambda \frac{\partial}{\partial \lambda} \int \frac{d^{2} z}{\lambda\left(z_{1}+z_{2}\right)^{3}} e^{\lambda\left(\frac{z_{1} z_{2}}{z_{1}+z_{2}} p^{2}-m_{f}^{2}\left(z_{1}+z_{2}\right)\right)} .
$$

If we now scale back $z_{i} \rightarrow x_{i} / \lambda$ we observe that the integral becomes $\lambda$-independent and thus its derivative with respect to $\lambda$ vanishes, namely

$$
\mathcal{I}=-\lambda \frac{\partial}{\partial \lambda} \int \frac{d^{2} x}{\left(x_{1}+x_{2}\right)^{3}} e^{\frac{x_{1} x_{2}}{x_{1}+x_{2}} p^{2}-m_{f}^{2}\left(x_{1}+x_{2}\right)}=0 .
$$

Therefore, the vacuum polarization tensor becomes

$$
\begin{aligned}
i \Pi^{\mu \nu}\left(p,\left|q_{f} B\right| \rightarrow 0\right) \\
=-\frac{i}{8 \pi^{2}} g^{2} \int d^{2} x \exp \left[\frac{x_{1} x_{2}}{x_{1}+x_{2}} p^{2}-m_{f}^{2}\left(x_{1}+x_{2}\right)\right] \\
\quad \times \frac{2 x_{1} x_{2}}{\left(x_{1}+x_{2}\right)^{4}} p^{2}\left(g^{\mu \nu}-\frac{p^{\mu} p^{\nu}}{p^{2}}\right) .
\end{aligned}
$$

Notice that Eq. (33) can also be written as

$$
\begin{aligned}
i \Pi^{\mu \nu}\left(p,\left|q_{f} B\right| \rightarrow 0\right) \\
=-\frac{i}{8 \pi^{2}} g^{2} \int d^{2} x \exp \left[\frac{x_{1} x_{2}}{x_{1}+x_{2}} p^{2}-m_{f}^{2}\left(x_{1}+x_{2}\right)\right] \\
\quad \times \frac{2 x_{1} x_{2}}{\left(x_{1}+x_{2}\right)^{4}} p^{2}\left(\mathcal{P}_{0}^{\mu \nu}+\mathcal{P}_{\|}^{\mu \nu}+\mathcal{P}_{\perp}^{\mu \nu}\right)
\end{aligned}
$$

where $\mathcal{P}_{0}^{\mu \nu}, \mathcal{P}_{\|}^{\mu \nu}$ and $\mathcal{P}_{\perp}^{\mu \nu}$ are given by Eqs. (16)-(18).

A similar argument is valid for a nonvanishing magnetic field. This means that the coefficients $A_{1}$ and $A_{2}$, in Eqs. (26) and (27), respectively, do not contribute to $\Pi^{\mu \nu}$, since they vanish. The systematic evaluation of these terms is shown in Appendix C. Thus, the full polarization tensor with the desired physical properties is given by

$$
\begin{aligned}
i \Pi^{\mu \nu}= & -\frac{i}{8 \pi^{2}} g^{2} \int d^{2} x f_{0}\left(x_{1}, x_{2}\right) \\
& \times\left[\Pi_{\|}\left(x_{1}, x_{2}\right) \mathcal{P}_{\|}^{\mu \nu}+\Pi_{\perp}\left(x_{1}, x_{2}\right) \mathcal{P}_{\perp}^{\mu \nu}+\Pi_{0}\left(x_{1}, x_{2}\right) \mathcal{P}_{0}^{\mu \nu}\right],
\end{aligned}
$$

where $\Pi_{\|}, \Pi_{\perp}$ and $\Pi_{0}$ are given by Eqs. (23)-(25), respectively.

To cancel the vacuum piece, we subtract from Eq. (35) the contribution from Eq. (33). Therefore, the finite, magnetic field-dependent part of the gluon polarization tensor is explicitly given by 


$$
\begin{aligned}
i \Pi^{\mu \nu}= & -\frac{i\left|q_{f} B\right|}{8 \pi^{2}} g^{2} \int \frac{d^{2} x}{\left(x_{1}+x_{2}\right)^{2}} \exp \left[\frac{x_{1} x_{2}}{x_{1}+x_{2}} p_{\|}^{2}-m_{f}^{2}\left(x_{1}+x_{2}\right)\right] \exp \left[-\frac{\tanh \left(\left|q_{f} B\right| x_{1}\right) \tanh \left(\left|q_{f} B\right| x_{2}\right)}{\tanh \left(\left|q_{f} B\right| x_{1}\right)+\tanh \left(\left|q_{f} B\right| x_{2}\right)} \frac{p_{\perp}^{2}}{\left|q_{f} B\right|}\right] \\
& \times\left\{\left[\frac{2 x_{1} x_{2} \operatorname{coth}\left[\left|q_{f} B\right|\left(x_{1}+x_{2}\right)\right]}{\left(x_{1}+x_{2}\right)} p_{\|}^{2}-\frac{x_{1} \sinh \left(2\left|q_{f} B\right| x_{2}\right)}{\sinh ^{2}\left[\left|q_{f} B\right|\left(x_{1}+x_{2}\right)\right]} p_{\perp}^{2}-\tilde{\Pi}\left(x_{1}, x_{2}\right)\right] \mathcal{P}_{\|}^{\mu \nu}\right. \\
& +\left[\frac{x_{1} \sinh \left(2\left|q_{f} B\right| x_{2}\right)}{\sinh ^{2}\left[\left|q_{f} B\right|\left(x_{1}+x_{2}\right)\right]} p_{\|}^{2}-\frac{2\left(x_{1}+x_{2}\right) \sinh \left(\left|q_{f} B\right| x_{1}\right) \sinh \left(\left|q_{f} B\right| x_{2}\right)}{\sinh ^{3}\left[\left|q_{f} B\right|\left(x_{1}+x_{2}\right)\right]} p_{\perp}^{2}-\tilde{\Pi}\left(x_{1}, x_{2}\right)\right] \mathcal{P}_{\perp}^{\mu \nu} \\
& \left.+\left[\frac{x_{1} \sinh \left(2\left|q_{f} B\right| x_{2}\right)}{\sinh ^{2}\left[\left|q_{f} B\right|\left(x_{1}+x_{2}\right)\right]} p^{2}-\tilde{\Pi}\left(x_{1}, x_{2}\right)\right] \mathcal{P}_{0}^{\mu \nu}\right\},
\end{aligned}
$$

where

$$
\tilde{\Pi}\left(x_{1}, x_{2}\right)=\frac{2 p^{2}}{\left|q_{f} B\right|} \frac{x_{1} x_{2}}{\left(x_{1}+x_{2}\right)^{2}} \exp \left(\frac{\tanh \left(\left|q_{f} B\right| x_{1}\right) \tanh \left(\left|q_{f} B\right| x_{2}\right)}{\tanh \left(\left|q_{f} B\right| x_{1}\right)+\tanh \left(\left|q_{f} B\right| x_{2}\right)} \frac{p_{\perp}^{2}}{\left|q_{f} B\right|}-\frac{x_{1} x_{2}}{x_{1}+x_{2}} p_{\perp}^{2}\right),
$$

and we have used the symmetry of the integral under the exchange $x_{1} \leftrightarrow x_{2}$. In order to check the validity of the above expression, we proceed to study its limits in the strong and weak magnetic field cases.

\section{STRONG FIELD LIMIT}

In order to study the strong field limit, let us first introduce the dimensionless variables

$$
y_{i} \equiv m_{f}^{2} x_{i}, \quad \rho_{\|, \perp}^{2} \equiv \frac{p_{\|, \perp}^{2}}{m_{f}^{2}}, \quad \mathcal{B} \equiv \frac{\left|q_{f} B\right|}{m_{f}^{2}}
$$

and the new variables $s$ and $y$ related to $y_{1}$ and $y_{2}$ by

$$
y_{1} \equiv s(1-y), \quad y_{2} \equiv s y,
$$

so that Eq. (35) becomes

$$
\begin{aligned}
i \Pi^{\mu \nu}= & -\frac{i g^{2} m_{f}^{2}}{8 \pi^{2}} \int_{0}^{1} d y \int_{0}^{\infty} d s \exp \left[s\left(y(1-y) \rho_{\|}^{2}-1\right)\right] \exp \left[-\frac{\cosh (\mathcal{B} s)-\cosh [\mathcal{B} s(2 y-1)]}{2 \sinh (\mathcal{B} s)} \frac{\rho_{\perp}^{2}}{\mathcal{B}}\right] \\
& \times\left\{\mathcal{B}\left[2 y(1-y) \operatorname{coth}(\mathcal{B} s) \rho_{\|}^{2}-\frac{(1-y) \sinh (2 \mathcal{B} s y)}{\sinh ^{2}(\mathcal{B} s)} \rho_{\perp}^{2}\right] \mathcal{P}_{\|}^{\mu \nu}\right. \\
& \left.+\mathcal{B}\left[\frac{(1-y) \sinh (2 \mathcal{B} s y)}{\sinh ^{2}(\mathcal{B} s)} \rho_{\|}^{2}-\frac{\cosh (\mathcal{B} s)-\cosh [\mathcal{B} s(2 y-1)]}{\sinh ^{3}(\mathcal{B} s)} \rho_{\perp}^{2}\right] \mathcal{P}_{\perp}^{\mu \nu}+\frac{(1-y) \mathcal{B} \sinh (2 \mathcal{B} s y)}{\sinh ^{2}(\mathcal{B} s)} \rho^{2} \mathcal{P}_{0}^{\mu \nu}\right\} .
\end{aligned}
$$

Note that in the strong field limit

$$
\begin{aligned}
\mathcal{B} \operatorname{coth}(\mathcal{B} s) & \sim \mathcal{B}, \\
\frac{\mathcal{B} \sinh (2 \mathcal{B} s y)}{2 \sinh ^{2}(\mathcal{B} s)} & \sim 0, \\
\frac{\cosh (\mathcal{B} s)-\cosh [\mathcal{B} s(2 y-1)]}{2 \sinh (\mathcal{B} s)} & \sim \frac{1}{2 \mathcal{B}}
\end{aligned}
$$

which hold for all $s$ and $0<y<1$. Therefore

$$
i \Pi^{\mu \nu}=-\frac{i g^{2} m_{f}^{2} \mathcal{B} \rho_{\|}^{2}}{4 \pi^{2}} e^{-\rho_{\perp}^{2} / 2 \mathcal{B}} \int_{0}^{1} d y y(1-y) \int_{0}^{\infty} d s \exp \left[s\left(y(1-y) \rho_{\|}^{2}-1\right)\right] \mathcal{P}_{\|}^{\mu \nu} .
$$




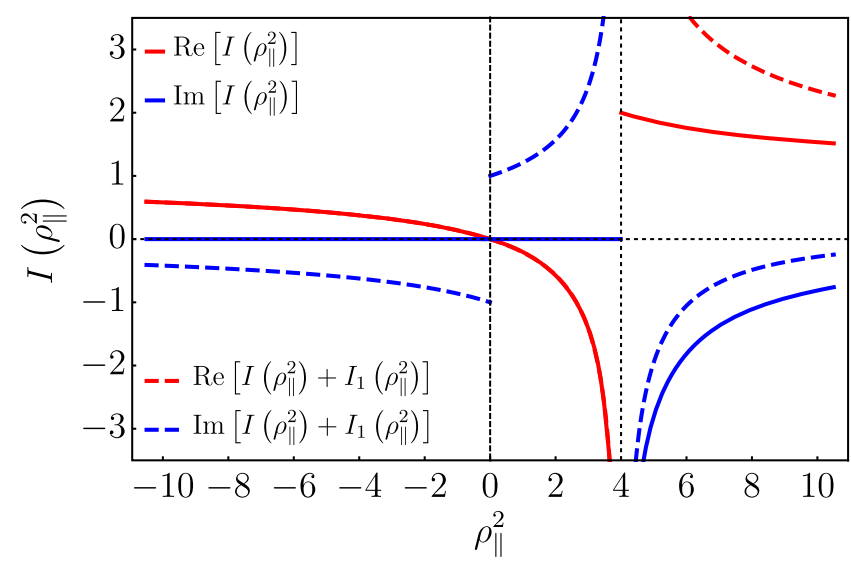

FIG. 2. Real and imaginary parts of the function $I\left(\rho_{\|}^{2}\right)$ defined in Eq. (42). Notice the discontinuity at the threshold value $\rho_{\|}=4$ or equivalently at $p_{\|}^{2}=4 m_{f}^{2}$. The result including the spurious contribution of $I_{1}\left(\rho_{\|}^{2}\right)$ from Eq. (43) is also plotted for comparison.

For the kinematical region such that $y(1-y) \rho_{\|}^{2}<1$, the integration over $s$ can be performed, yielding

$$
\begin{aligned}
i \Pi^{\mu \nu} & =\frac{i g^{2} m_{f}^{2} \mathcal{B}}{4 \pi^{2}} e^{-\rho_{\perp}^{2} / 2 \mathcal{B}} \int_{0}^{1} d y \frac{y(1-y)}{y(1-y)-\rho_{\|}^{-2}} \mathcal{P}_{\|}^{\mu \nu} \\
& \equiv \frac{i g^{2} m_{f}^{2} \mathcal{B}}{4 \pi^{2}} e^{-\rho_{\perp}^{2} / 2 \mathcal{B}} I\left(\rho_{\|}^{2}\right) \mathcal{P}_{\|}^{\mu \nu}
\end{aligned}
$$

which coincides with the result obtained in Refs. [14-16] where the gluon polarization tensor is computed by considering only the contribution from the LLL.

Figure 2 shows the real and imaginary parts of $I\left(\rho_{\|}^{2}\right)$ compared with the result obtained when the contribution of $A_{1}$, according to Eq. (26) is considered. From Eq. (28a) the spurious term contributes with

$$
i \Pi^{\mu \nu}=\frac{i g^{2} m_{f}^{2} \mathcal{B}}{4 \pi^{2}} e^{-\rho_{\perp}^{2} / 2 \mathcal{B}}\left[I\left(\rho_{\|}^{2}\right)+I_{1}\left(\rho_{\|}^{2}\right)\right] \mathcal{P}_{\|}^{\mu \nu},
$$

where

$$
\begin{aligned}
I_{1}(x) & =\int_{0}^{1} d y \frac{1}{y(1-y) x-1} \\
& =-\frac{4}{\sqrt{x(x-4)}} \arctan \left(\frac{\sqrt{x}}{\sqrt{4-x}}\right) .
\end{aligned}
$$

From Fig. 2 a discontinuity at the threshold value $\rho_{\|}^{2}=4$ or equivalently at $p_{\|}^{2}=4 m_{f}^{2}$ can be identified. As the figure indicates, the spurious contribution generates an unphysical threshold at $\rho_{\|}^{2}=0$, which cannot be identified with a fermion-pair creation. Notice also that Eq. (36) implies the existence of an infinite sequence of momentum thresholds when the external gluon momentum becomes resonant with twice the quark/antiquark magnetic mass, whose square is defined as $m_{(B) f}^{2}=m_{f}^{2}+2 n\left|q_{f} B\right|$. The threshold corresponds to the value of the longitudinal momentum squared for the creation of a quark-antiquark pair, each particle having a magnetic mass corresponding to the given Landau level.

These thresholds can be obtained from our calculation by concentrating on the conditions where the hyperbolic functions become divergent. For these purposes let us examine the term proportional to $\operatorname{coth}(\mathcal{B} s)$ in Eq. (39)

$$
\begin{aligned}
\mathcal{K}= & \int_{0}^{1} d y \int_{0}^{\infty} d s y(1-y) \mathcal{B} \operatorname{coth}(\mathcal{B} s) \\
& \times \exp \left[s\left(y(1-y) \rho_{\|}^{2}-1\right)\right] \\
& \times \exp \left[-\frac{\cosh (\mathcal{B} s)-\cosh [\mathcal{B} s(2 y-1)]}{2 \sinh (\mathcal{B} s)} \frac{\rho_{\perp}^{2}}{\mathcal{B}}\right] .
\end{aligned}
$$

Notice that if $\mathcal{B} \gg 1$

$$
\begin{aligned}
& \mathcal{B} \operatorname{coth}(\mathcal{B} s) \exp \left[-\frac{\cosh (\mathcal{B} s)-\cosh [\mathcal{B} s(2 y-1)]}{2 \sinh (\mathcal{B} s)} \frac{\rho_{\perp}^{2}}{\mathcal{B}}\right] \\
& =\mathcal{B} \frac{1+e^{-2 \mathcal{B} s}}{1-e^{-2 \mathcal{B} s}} \exp \left\{\frac{-1-e^{-2 \mathcal{B} s}+e^{-2 \mathcal{B} s(y-1)}+e^{-2 \mathcal{B} s y}}{2 \mathcal{B}\left(1-e^{-2 \mathcal{B} s}\right)} \rho_{\perp}^{2}\right\} \\
& \approx \mathcal{B} \frac{1+e^{-2 \mathcal{B} s}}{1-e^{-2 \mathcal{B} s}}+\mathcal{O}\left(\rho_{\perp}^{2}\right) .
\end{aligned}
$$

Using that

$$
\frac{1}{1-e^{-2 \mathcal{B} s}}=\sum_{n=0}^{\infty} e^{-2 n \mathcal{B} s},
$$

we can write

$$
\frac{1+e^{-2 \mathcal{B} s}}{1-e^{-2 \mathcal{B} s}}=1+2 \sum_{n=1}^{\infty} e^{-2 n \mathcal{B} s}
$$

so that, the dominant term in Eq. (45) is given by

$$
\begin{aligned}
\mathcal{K}= & \mathcal{B} \int_{0}^{1} d y \int_{0}^{\infty} d s y(1-y)\left\{\exp \left[s\left(y(1-y) \rho_{\|}^{2}-1\right)\right]\right. \\
& \left.+2 \sum_{n=1}^{\infty} \exp \left[s\left(y(1-y) \rho_{\|}^{2}-2 n \mathcal{B}-1\right)\right]\right\} \\
= & \frac{\mathcal{B}}{\rho_{\|}^{2}} I\left(\rho_{\|}^{2}\right)+8 \mathcal{B} J\left(\rho_{\|}^{2}\right),
\end{aligned}
$$

where $I(x)$ is defined in Eq. (42) and 


$$
J(x) \equiv-\sum_{n=1}^{\infty} \frac{\arctan \left(\frac{\sqrt{x}}{\sqrt{4(2 n \mathcal{B}+1)-x}}\right)}{\sqrt{x[4(2 n \mathcal{B}+1)-x]}} .
$$

In this way, the resonant behavior of the thresholds is explicit: the gluon polarization tensor has divergences when its momentum reaches the value $p_{\|}^{2}=4 m_{(B n) f}^{2}$, where $n$ labels each of the Landau levels. In other words, the creation of quark-antiquark pairs is allowed when the gluon momentum is large enough to generate not only the inertial mass of the pair but rather the magnetic mass, induced by the magnetized medium. Figure 3 shows several thresholds of the function $J\left(\rho_{\|}^{2}\right)$ in a broad range of $\rho_{\|}^{2}$ for a maximum value of $n, n_{\max }=100$. The same argument is valid for all terms in Eq. (39) given that its dominant contribution is given by a power of the series in Eq. (47).

\section{WEAK FIELD LIMIT}

Let us study the case where the field satisfies the hierarchy of energy scales $|e B|<m_{f}^{2}$. We call this the

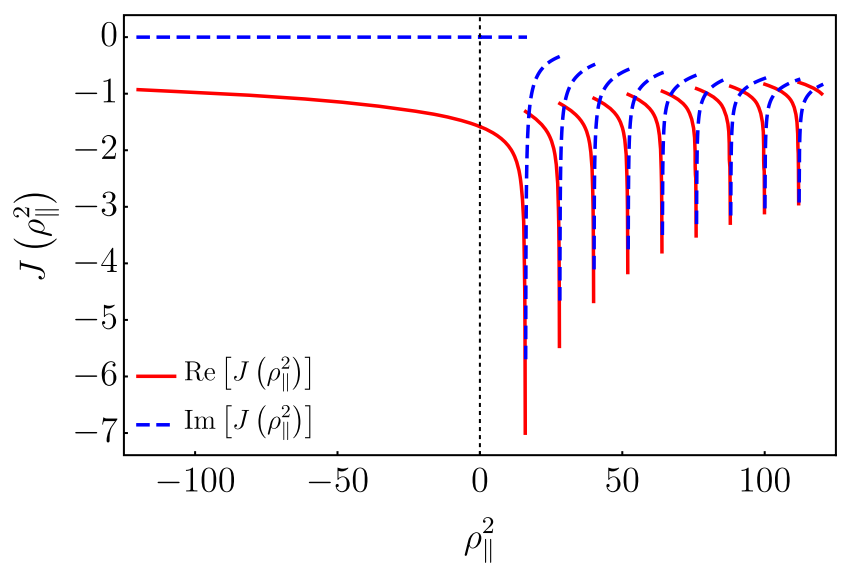

FIG. 3. Real and imaginary parts of the function $J\left(\rho_{\|}^{2}\right)$ defined in Eq. (50) for $\mathcal{B}=1.5$ and added up to $n_{\max }=100$. Notice the emergence of different resonant thresholds when the quark magnetic mass includes consecutive Landau levels.

weak field limit. For this purpose, we can perform a power series of Eq. (39) around $\mathcal{B}=0$ to obtain

$$
\begin{aligned}
i \Pi^{\mu \nu}= & -\frac{i g^{2} m_{f}^{2}}{8 \pi^{2}} \int_{0}^{1} d y \int_{0}^{\infty} d s\left\{\left[\frac{2 y(1-y)}{s} \rho^{2}+\frac{2 s y(1-y)}{3}\left[s y^{2}(1-y)^{2} \rho_{\perp}^{2} \rho^{2}+2\left(1-y^{2}\right) \rho_{\perp}^{2}+\rho^{2}\right] \mathcal{B}^{2}\right] \mathcal{P}_{\|}^{\mu \nu}\right. \\
& +\left[\frac{2 y(1-y)}{s} \rho^{2}+\frac{2 s y(1-y)}{3}\left[s y^{2}(1-y)^{2} \rho_{\perp}^{2} \rho^{2}+(1+y) \rho_{\perp}^{2}-\left(1-y^{2}\right) \rho_{\|}^{2}+y^{2} \rho^{2}\right] \mathcal{B}^{2}\right] \mathcal{P}_{\perp}^{\mu \nu} \\
& \left.+\left[\frac{2 y(1-y)}{s} \rho^{2}+\frac{2 s y(1-y)}{3} \rho^{2}\left[s y^{2}(1-y)^{2} \rho_{\perp}^{2}+2 y^{2}-1\right] \mathcal{B}^{2}\right] \mathcal{P}_{0}^{\mu \nu}\right\} \exp \left[s\left(y(1-y) \rho^{2}-1\right)\right],
\end{aligned}
$$

where the vacuum contribution of Eq. (34) can be identified as

$$
i \Pi^{\mu \nu}\left(\rho^{2}, \mathcal{B} \rightarrow 0\right)=-\frac{i g^{2} m_{f}^{2}}{8 \pi^{2}} \int_{0}^{1} d y \int_{0}^{\infty} d s \exp \left[s\left(y(1-y) \rho^{2}-1\right)\right] \frac{2 y(1-y)}{s} \rho^{2}\left(\mathcal{P}_{\|}^{\mu \nu}+\mathcal{P}_{\perp}^{\mu \nu}+\mathcal{P}_{0}^{\mu \nu}\right) .
$$

Subtracting this contribution, we are left with the $\mathcal{B}$-dependent part. The integrations over $s$ and $y$ can be performed analytically, so that

$$
i \Pi_{\mathrm{Weak} \mathcal{B}}^{\mu \nu}=-\frac{i g^{2} m_{f}^{2} \mathcal{B}^{2}}{6 \pi^{2}}\left[\hat{\Pi}_{\|}\left(\rho^{2}\right) \mathcal{P}_{\|}^{\mu \nu}+\hat{\Pi}_{\perp}\left(\rho^{2}\right) \mathcal{P}_{\perp}^{\mu \nu}+\hat{\Pi}_{0}\left(\rho^{2}\right) \mathcal{P}_{0}^{\mu \nu}\right]
$$

where

$$
\begin{aligned}
& \hat{\Pi}_{\|}=\frac{1}{4-\rho^{2}}\left[\left(12 \frac{10+\left(\rho^{2}-6\right) \rho^{2}}{\left(4-\rho^{2}\right)^{3 / 2}\left(\rho^{2}\right)^{5 / 2}} \rho_{\perp}^{2}+2 \frac{\rho^{2}\left(\rho^{2}+2\right)-12}{\sqrt{4-\rho^{2}}\left(\rho^{2}\right)^{5 / 2}} \rho_{\perp}^{2}+2 \frac{\left(\rho^{2}-2\right) \sqrt{\left(4-\rho^{2}\right) \rho^{2}}}{\left(4-\rho^{2}\right) \rho^{2}}\right) \arctan \left(\frac{\sqrt{\rho^{2}}}{\sqrt{4-\rho^{2}}}\right)\right. \\
& \left.-\frac{\left(\rho^{2}-10\right)\left(\rho^{2}-3\right)}{4-\rho^{2}} \frac{\rho_{\perp}^{2}}{\rho^{4}}+\frac{6 \rho_{\perp}^{2}}{\rho^{2}}+1\right] \\
& \hat{\Pi}_{\perp}=\frac{1}{4-\rho^{2}}\left[\left(12 \frac{10+\left(\rho^{2}-6\right) \rho^{2}}{\left(4-\rho^{2}\right)^{3 / 2}\left(\rho^{2}\right)^{5 / 2}} \rho_{\perp}^{2}+3 \frac{\left(\rho^{2}-2\right) \sqrt{\left(4-\rho^{2}\right) \rho^{2}}}{\left(4-\rho^{2}\right) \rho^{4}} \rho_{\perp}^{2}-\frac{\rho^{2}\left(\rho^{2}+2\right)-12}{\sqrt{4-\rho^{2}}\left(\rho^{2}\right)^{5 / 2}} \rho_{\|}^{2}+\frac{12+\left(\rho^{2}-6\right) \rho^{2}}{\sqrt{4-\rho^{2}}\left(\rho^{2}\right)^{3 / 2}}\right) \arctan \left(\frac{\sqrt{\rho^{2}}}{\sqrt{4-\rho^{2}}}\right)\right. \\
& \left.-\frac{\left(\rho^{2}-10\right)\left(\rho^{2}-3\right)}{4-\rho^{2}} \frac{\rho_{\perp}^{2}}{\rho^{4}}+\frac{3 \rho_{\perp}^{2}}{2 \rho^{2}}-\frac{3 \rho_{\|}^{2}}{\rho^{4}}+\frac{\left(\rho^{2}-3\right) \sqrt{\left(4-\rho^{2}\right) \rho^{2}}}{\sqrt{4-\rho^{2}}\left(\rho^{2}\right)^{3 / 2}}\right],
\end{aligned}
$$




$$
\begin{aligned}
\hat{\Pi}_{0}= & \frac{1}{4-\rho^{2}}\left[\left(12 \frac{10+\left(\rho^{2}-6\right) \rho^{2}}{\left(4-\rho^{2}\right)^{3 / 2}\left(\rho^{2}\right)^{5 / 2}} \rho_{\perp}^{2}+2 \frac{12+\left(\rho^{2}-6\right) \rho^{2}}{\sqrt{4-\rho^{2}}\left(\rho^{2}\right)^{3 / 2}}-2 \frac{\left(\rho^{2}-2\right) \sqrt{\left(4-\rho^{2}\right) \rho^{2}}}{\left(4-\rho^{2}\right) \rho^{2}}\right) \arctan \left(\frac{\sqrt{\rho^{2}}}{\sqrt{4-\rho^{2}}}\right)\right. \\
& \left.-\frac{\left(\rho^{2}-10\right)\left(\rho^{2}-3\right)}{4-\rho^{2}} \frac{\rho_{\perp}^{2}}{\rho^{4}}+2 \frac{\left(\rho^{2}-3\right) \sqrt{\left(4-\rho^{2}\right) \rho^{2}}}{\sqrt{4-\rho^{2}}\left(\rho^{2}\right)^{3 / 2}}-1\right] .
\end{aligned}
$$

The coefficients $\hat{\Pi}_{\|}, \hat{\Pi}_{\perp}$ and $\hat{\Pi}_{0}$ consist of real and imaginary parts. The imaginary parts can be obtained from the corresponding real parts from the Kramers-Kronig relations. With the notation $\omega \equiv \rho_{0}$, we have
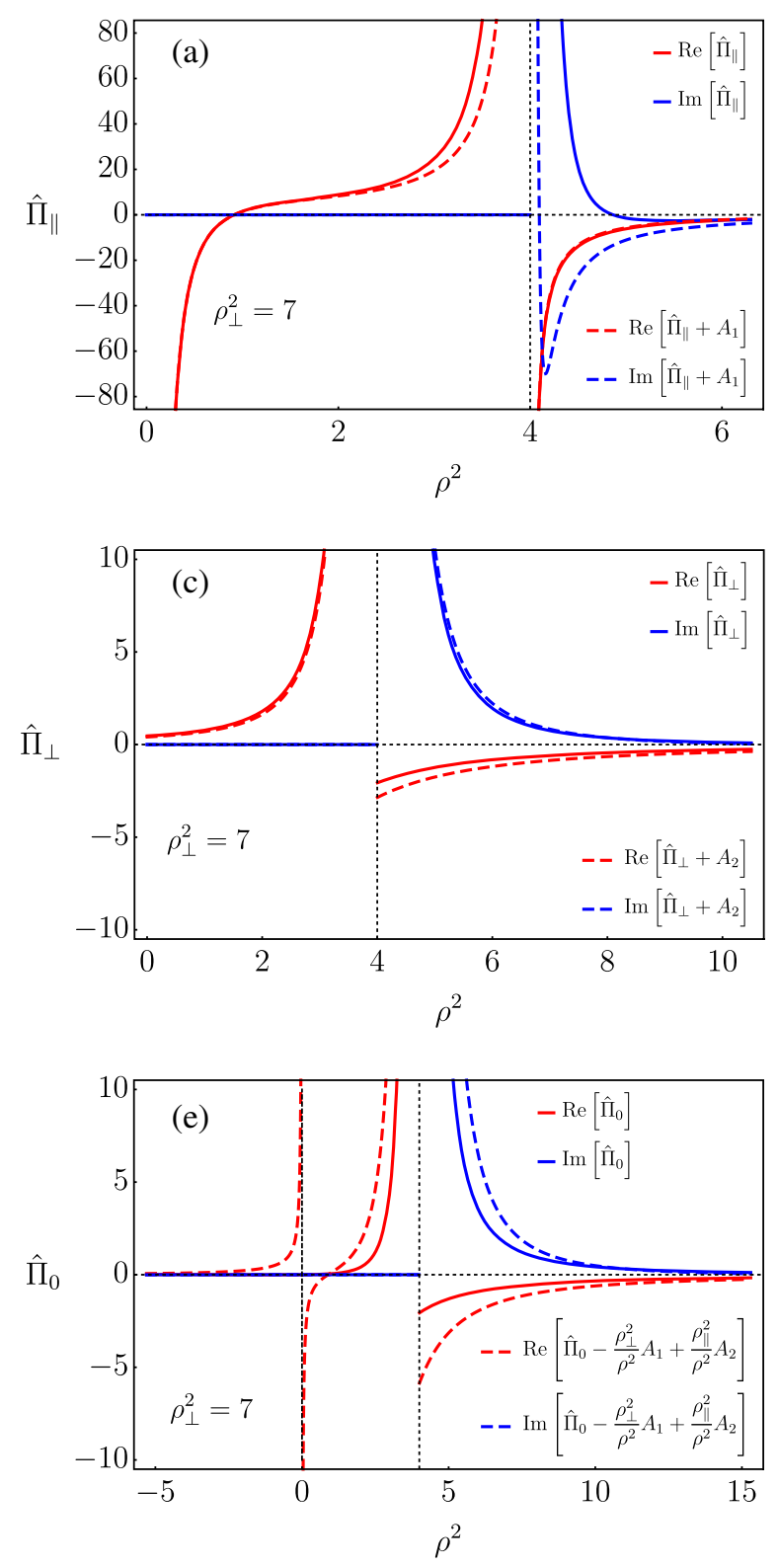

$$
\operatorname{Im} \Pi^{\mu \nu}(\omega)=-\frac{1}{\pi} \mathcal{P} \int_{-\infty}^{+\infty} \frac{\operatorname{Re} \Pi^{\mu \nu}\left(\omega^{\prime}\right)}{\omega^{\prime}-\omega} d \omega^{\prime}
$$

where $\mathcal{P}$ is the principal value. Examples of these coefficients as functions of $\rho_{\|}^{2}$, for various values of $\rho_{\perp}^{2}$ are
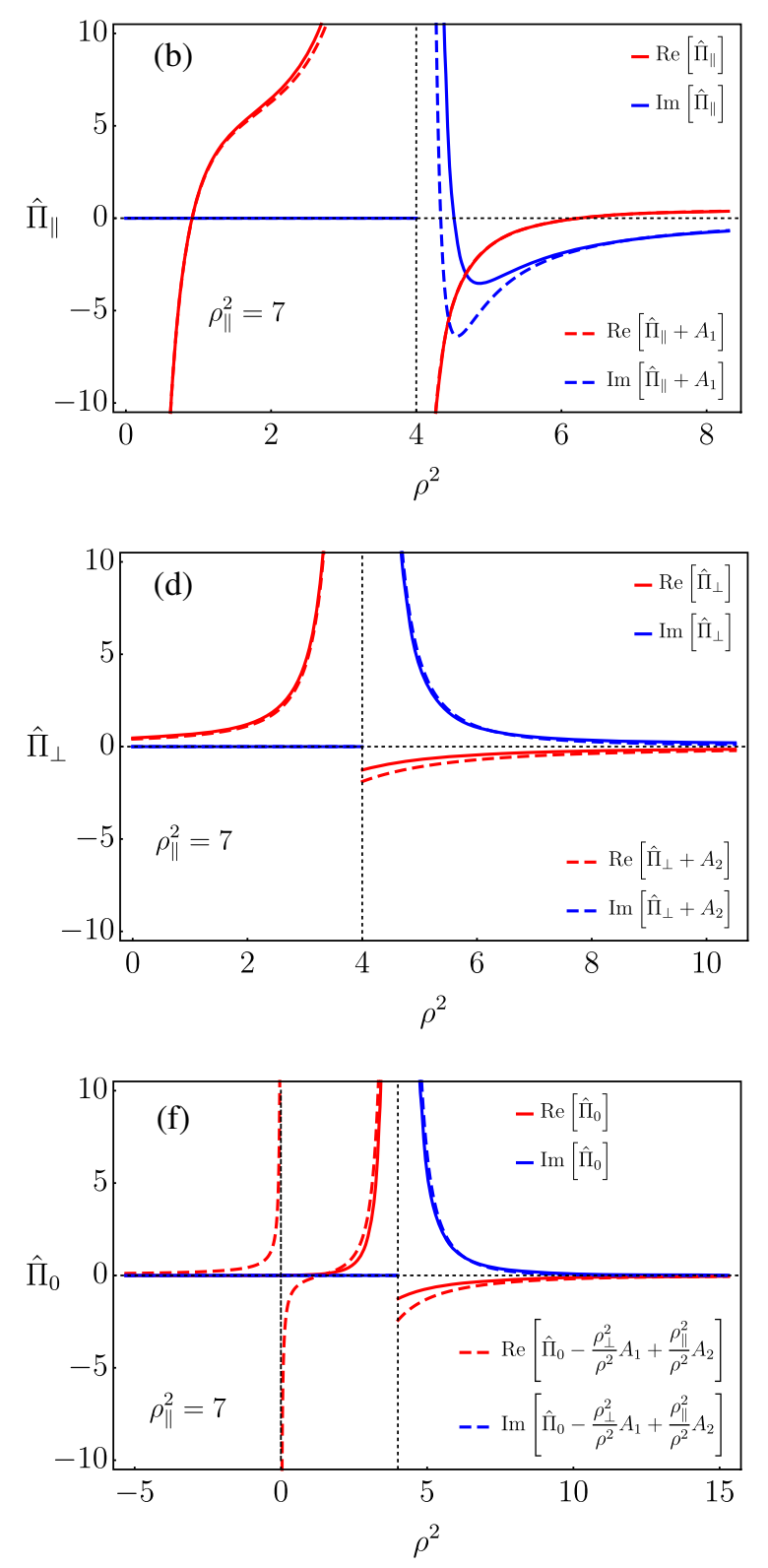

FIG. 4. Real and imaginary parts of the coefficients $\hat{\Pi}_{\|}, \hat{\Pi}_{\perp}$ and $\hat{\Pi}_{0}$ from Eqs. (54)-(56) as functions of $\rho^{2}$ for fixed values of $\rho_{\|}^{2}$ and $\rho_{\|}^{2}$. For comparison, these coefficients are also plotted including he spurious contributions from $A_{1}$ and $A_{2}$, given by Eqs. (28a)-(28c). Notice that for the chosen kinematical range for $\rho^{2}$, the threshold appears at $\rho^{2}=4$ or equivalently at $p^{2}=4 m_{f}^{2}$, whereas the spurious terms contain unphysical thresholds at $\rho^{2}=0$. 
shown in Fig. 4. For comparison, these coefficients are also plotted including he spurious contributions from $A_{1}$ and $A_{2}$ given by Eqs. (28a)-(28c). Notice the appearance of unphysical thresholds at $\rho^{2}=0$ as well as large deviations from the correct functional behavior of the coefficients of the tensor structures.

\section{RESULTS, DISCUSSION AND CONCLUSIONS}

The results of this work can be used to study birefringence of the gluon polarization in a magnetized medium. Recall that birefringence is the optical property exhibited by a material whose refractive index depends on the polarization and propagation direction of light. In solid-state, crystals with noncubic lattice symmetry show birefringence, with calcite being a typical and historical example. The simplest type of birefringence corresponds to the so-called uniaxial type, where a single direction governs the optical anysotropy while all the other directions orthogonal to it are optically equivalent. Thus, rotations of the crystal with respect to this axis leave the optical response invariant. On the other hand, a material that is otherwise optically isotropic can manifest birefringence under the presence of external agents, such as strain and, more importantly, an external magnetic field. This last case is often called Faraday effect [24]. An analogous situation is studied in the context of high-energy physics, particularly in QED under the presence of static magnetic or electric fields, where the index of refraction depends on the photon polarization state.

Despite the absence of an underlying discrete symmetry as in crystalline materials, the presence of these static fields is often sufficient to induce optical birefringence under certain conditions, which in this context is called vacuum birefringence. This effect has been extensively studied theoretically $[17,25]$. Moreover, recent experimental evidence for this phenomenon has been provided from astronomical observations of neutron stars, where intense magnetic fields are present [26].

In QED, the microscopic mechanism behind the effect are the vacuum fluctuations due to the spontaneous emergence of virtual electron-positron pairs that act as dipoles, in analogy with dielectric crystals. In the absence of external fields, Lorentz invariance ensures an isotropic optical response. However, when a static electric or magnetic field is present, Lorentz invariance is broken and an anisotropic optical response is triggered. In particular, when a magnetic field is responsible for the effect, the virtual fermion pair exists in general in a combination of Landau levels.

In this work, we show that vacuum birefringence arises also for gluons in QCD, where the virtual fermion-antifermion pairs correspond to quark-antiquark pairs that play the same role as electron-positron pairs in QED. Just as in
QED, the QCD version of the phenomenon necessarily implies the existence of an infinite sequence of momentum thresholds, that correspond to the condition where the external gluon momentum is resonant with the magnetic mass of a pair occupying a given Landau level, which are successively occupied by the pair of virtual quarks participating in the process.

We have presented a method to compute the one-loop magnetic correction to the gluon polarization tensor starting from the Landau-level representation of the quark propagator in the presence of an external magnetic field. With suitable transformations, we have shown that this representation can be converted into the expression for the one-loop polarization tensor equivalent to the one obtained starting from Schwinger's proper time representation of the quark propagators. We have shown that the general expression contains the vacuum contribution that can be isolated from the zero-field limit for finite gluon momentum. This can be achieved only when the whole sum over levels is performed. Therefore, calculations that resort to partial sums over Landau levels run the risk to mask the vacuum contributions and distort the result. An important observation is that, the general tensor structure for the gluon polarization contains two spurious terms that do not satisfy the transversality properties. We have shown that, in analogy with the case in vacuum, these terms have vanishing coefficients and thus do not contribute to the polarization tensor, as expected. Nevertheless, strictly speaking, this result requires that the $i \epsilon$ term in the quark propagators is not taken to zero, for otherwise the integrals representing the coefficients of the spurious terms are not oscillatory and the areas above and below the $x_{i}$ axis cannot cancel. However, as it is customary, this term is only kept implicit in the calculation and is only brought back, for instance, when computing the real and imaginary parts of the final result. Thus, if the coefficients of the spurious terms are not shown to vanish and care is not taken when computing the coefficients upon projection onto the chosen basis, these can give contributions that are not correct, as we have shown. In order to check the validity of the expressions thus found, we have shown that the strong field limit obtained from our approach reproduces a well-established result. The results of this work can be used to study the conditions for gluons to equilibrate with a magnetized medium, for example during the early stages of a relativistic heavy-ion collision. This is work in progress and it will be reported elsewhere.

\section{ACKNOWLEDGMENTS}

The authors are thankful to L. A. Hernández and R. Zamora for useful conversations. Support for this work was received in part by UNAM-DGPA-PAPIIT Grant No. IG100219 and by Consejo Nacional de Ciencia y 
Tecnología Grant No. 256494. E. M. acknowledges support from FONDECYT (Chile) under Grant No. 1190361. M. L. acknowledges support from FONDECYT (Chile) under Grants No. 1170107 and No. 1190192 and from Conicyt/ PIA/Basal (Chile) Grant No. FB0821.

\section{APPENDIX A: DERIVATION OF EQS. (7)}

Let us begin from the general expression of the gluon polarization tensor of Eq. (1): $i \Pi_{(a b)}^{\mu \nu}=-\frac{1}{2} \int \frac{d^{4} k}{(2 \pi)^{4}} \operatorname{Tr}\left\{i g t_{b} \gamma^{\nu} i S^{(n)}(k) i g t_{a} \gamma^{\mu} i S^{(m)}(q)\right\}+$ C.C.

The trace in the above expression involves two fermion propagator factors, each given by Eqs. (2) and (3). This product produces nine terms, that are explicitly given by

$$
\begin{aligned}
& t_{1}^{\mu \nu}=-g^{2} \sum_{n, m=0}^{\infty} \int \frac{d^{4} k}{(2 \pi)^{4}} \exp \left[-\frac{k_{\perp}^{2}+(k-p)_{\perp}^{2}}{\left|q_{f} B\right|}\right] \frac{(-1)^{n+m} L_{n}^{0}\left(\frac{2 k_{\perp}^{2}}{\left|q_{f} B\right|}\right) L_{m}^{0}\left[\frac{2(k-p)_{1}^{2}}{\left|q_{f} B\right|}\right]}{\left[k_{\|}^{2}-m_{f}^{2}-2 n\left|q_{f} B\right|\right]\left[(k-p)_{\|}^{2}-m_{f}^{2}-2 m\left|q_{f} B\right|\right]} \\
& \times \operatorname{Tr}\left\{\gamma^{\nu}\left(\not \not_{\|}+m_{f}\right) \mathcal{O}^{-} \gamma^{\mu}\left(\not \mathbb{H}_{\|}-\not \not_{\|}+m_{f}\right) \mathcal{O}^{-}\right\}+\text {C.C., } \\
& t_{2}^{\mu \nu}=g^{2} \sum_{n=0, m=1}^{\infty} \int \frac{d^{4} k}{(2 \pi)^{4}} \exp \left[-\frac{k_{\perp}^{2}+(k-p)_{\perp}^{2}}{\left|q_{f} B\right|}\right] \frac{(-1)^{n+m} L_{n}^{0}\left(\frac{2 k_{\perp}^{2}}{\left|q_{f} B\right|}\right) L_{m-1}^{0}\left[\frac{2(k-p)_{\perp}^{2}}{\left|q_{f} B\right|}\right]}{\left[k_{\|}^{2}-m_{f}^{2}-2 n\left|q_{f} B\right|\right]\left[(k-p)_{\|}^{2}-m_{f}^{2}-2 m\left|q_{f} B\right|\right]} \\
& \times \operatorname{Tr}\left\{\gamma^{\nu}\left(\not k_{\|}+m_{f}\right) \mathcal{O}^{-} \gamma^{\mu}\left(\not k_{\|}-\not \not_{\|}+m_{f}\right) \mathcal{O}^{+}\right\}+\text {C.C., } \\
& t_{3}^{\mu \nu}=-2 g^{2} \sum_{n=0, m=1}^{\infty} \int \frac{d^{4} k}{(2 \pi)^{4}} \exp \left[-\frac{k_{\perp}^{2}+(k-p)_{\perp}^{2}}{\left|q_{f} B\right|}\right] \frac{(-1)^{n+m} L_{n}^{0}\left(\frac{2 k_{\perp}^{2}}{\left|q_{f} B\right|}\right) L_{m-1}^{1}\left[\frac{2(k-p)_{\perp}^{2}}{\left|q_{f} B\right|}\right]}{\left[k_{\|}^{2}-m_{f}^{2}-2 n\left|q_{f} B\right|\right]\left[(k-p)_{\|}^{2}-m_{f}^{2}-2 m\left|q_{f} B\right|\right]} \\
& \times \operatorname{Tr}\left\{\gamma^{\nu}\left(\not k_{\|}+m_{f}\right) \mathcal{O}^{-} \gamma^{\mu}\left(\not k_{\perp}-\not p_{\perp}\right)\right\}+\text { C.C., } \\
& t_{4}^{\mu \nu}=g^{2} \sum_{n=1, m=0}^{\infty} \int \frac{d^{4} k}{(2 \pi)^{4}} \exp \left[-\frac{k_{\perp}^{2}+(k-p)_{\perp}^{2}}{\left|q_{f} B\right|}\right] \frac{(-1)^{n+m} L_{n-1}^{0}\left(\frac{2 k_{\perp}^{2}}{\left|q_{f} B\right|}\right) L_{m}^{0}\left[\frac{2(k-p)_{\perp}^{2}}{\left|q_{f} B\right|}\right]}{\left[k_{\|}^{2}-m_{f}^{2}-2 n\left|q_{f} B\right|\right]\left[(k-p)_{\|}^{2}-m_{f}^{2}-2 m\left|q_{f} B\right|\right]} \\
& \times \operatorname{Tr}\left\{\gamma^{\nu}\left(\not \|_{\|}+m_{f}\right) \mathcal{O}^{+} \gamma^{\mu}\left(\not k_{\|}-\not \not_{\|}+m_{f}\right) \mathcal{O}^{-}\right\}+\text {C.C., } \\
& t_{5}^{\mu \nu}=-g^{2} \sum_{n=1, m=1}^{\infty} \int \frac{d^{4} k}{(2 \pi)^{4}} \exp \left[-\frac{k_{\perp}^{2}+(k-p)_{\perp}^{2}}{\left|q_{f} B\right|}\right] \frac{(-1)^{n+m} L_{n-1}^{0}\left(\frac{2 k_{\perp}^{2}}{\left|q_{f} B\right|}\right) L_{m-1}^{0}\left[\frac{2(k-p)_{\perp}^{2}}{\left|q_{f} B\right|}\right]}{\left[k_{\|}^{2}-m_{f}^{2}-2 n\left|q_{f} B\right|\right]\left[(k-p)_{\|}^{2}-m_{f}^{2}-2 m\left|q_{f} B\right|\right]} \\
& \times \operatorname{Tr}\left\{\gamma^{\nu}\left(\not \mathbb{K}_{\|}+m_{f}\right) \mathcal{O}^{+} \gamma^{\mu}\left(\not ̈_{\|}-\not \not_{\|}+m_{f}\right) \mathcal{O}^{+}\right\}+\text {C.C., } \\
& t_{6}^{\mu \nu}=2 g^{2} \sum_{n=1, m=1}^{\infty} \int \frac{d^{4} k}{(2 \pi)^{4}} \exp \left[-\frac{k_{\perp}^{2}+(k-p)_{\perp}^{2}}{\left|q_{f} B\right|}\right] \frac{(-1)^{n+m} L_{n-1}^{0}\left(\frac{2 k_{\perp}^{2}}{\mid q_{f} B}\right) L_{m-1}^{1}\left[\frac{2(k-p)_{\perp}^{2}}{\left|q_{f} B\right|}\right]}{\left[k_{\|}^{2}-m_{f}^{2}-2 n\left|q_{f} B\right|\right]\left[(k-p)_{\|}^{2}-m_{f}^{2}-2 m\left|q_{f} B\right|\right]} \\
& \times \operatorname{Tr}\left\{\gamma^{\nu}\left(\not \not_{\|}+m_{f}\right) \mathcal{O}^{+} \gamma^{\mu}\left(\not \not_{\perp}-\not \not_{\perp}\right)\right\}+\text { C.C., } \\
& t_{7}^{\mu \nu}=-2 g^{2} \sum_{n=1, m=0}^{\infty} \int \frac{d^{4} k}{(2 \pi)^{4}} \exp \left[-\frac{k_{\perp}^{2}+(k-p)_{\perp}^{2}}{\left|q_{f} B\right|}\right] \frac{(-1)^{n+m} L_{n-1}^{1}\left(\frac{2 k_{\perp}^{2}}{\left|q_{f} B\right|}\right) L_{m}^{0}\left[\frac{2(k-p)_{\perp}^{2}}{\left|q_{f} B\right|}\right]}{\left[k_{\|}^{2}-m_{f}^{2}-2 n\left|q_{f} B\right|\right]\left[(k-p)_{\|}^{2}-m_{f}^{2}-2 m\left|q_{f} B\right|\right]} \\
& \times \operatorname{Tr}\left\{\gamma^{\nu} \not \not_{\perp} \gamma^{\mu}\left(\not k_{\|}-\not \not_{\|}+m_{f}\right) \mathcal{O}^{-}\right\}+\text {C.C., } \\
& t_{8}^{\mu \nu}=2 g^{2} \sum_{n=1, m=1}^{\infty} \int \frac{d^{4} k}{(2 \pi)^{4}} \exp \left[-\frac{k_{\perp}^{2}+(k-p)_{\perp}^{2}}{\left|q_{f} B\right|}\right] \frac{(-1)^{n+m} L_{n-1}^{1}\left(\frac{2 k_{\perp}^{2}}{\left|q_{f} B\right|}\right) L_{m-1}^{0}\left[\frac{2(k-p)^{2}}{\left|q_{f} B\right|}\right]}{\left[k_{\|}^{2}-m_{f}^{2}-2 n\left|q_{f} B\right|\right]\left[(k-p)_{\|}^{2}-m_{f}^{2}-2 m\left|q_{f} B\right|\right]} \\
& \times \operatorname{Tr}\left\{\gamma^{\nu} \not \not_{\perp} \gamma^{\mu}\left(\not \|_{\|}-\not \not_{\|}+m_{f}\right) \mathcal{O}^{+}\right\}+\text {C.C., }
\end{aligned}
$$


In order to perform the sum over Landau levels, we write the denominators introducing Schwinger parameters such that

$$
\frac{1}{y}=\int_{0}^{\infty} e^{-y x} d x
$$

We start with the expression given by Eq. (A2)

$$
\begin{aligned}
t_{1}^{\mu \nu}= & -g^{2} \int \frac{d^{4} k}{(2 \pi)^{4}} \exp \left[-\frac{k_{\perp}^{2}+(k-p)_{\perp}^{2}}{\left|q_{f} B\right|}\right] \operatorname{Tr}\left\{\gamma^{\nu}\left(\not k_{\|}+m_{f}\right) \mathcal{O}^{-} \gamma^{\mu}\left(\not k_{\|}-\not \not_{\|}+m_{f}\right) \mathcal{O}^{-}\right\} \\
& \times \sum_{n, m=0}^{\infty} \frac{(-1)^{n+m} L_{n}^{0}\left(\frac{2 k_{\perp}^{2}}{\left|q_{f} B\right|}\right) L_{m}^{0}\left[\frac{2(k-p)_{\perp}^{2}}{\left.\left|q_{f}^{2}-m_{f}^{2}-2 n\right| q_{f} B \mid\right]\left[(k-p)_{\|}^{2}-m_{f}^{2}-2 m\left|q_{f} B\right|\right]}\right.}{=}-g^{2} \int d^{2} x \int \frac{d^{4} k}{(2 \pi)^{4}} \exp \left[-\frac{k_{\perp}^{2}+(k-p)_{\perp}^{2}}{\left|q_{f} B\right|}\right] \operatorname{Tr}\left\{\gamma^{\nu}\left(\not k_{\|}+m_{f}\right) \mathcal{O}^{-} \gamma^{\mu}\left(\not k_{\|}-\not \boldsymbol{p}_{\|}+m_{f}\right) \mathcal{O}^{-}\right\} \\
& \times e^{\alpha\left(k_{\|}\right) x_{1}+\beta\left(k_{\|}\right) x_{2}} \sum_{n, m=0}^{\infty} r_{1}^{n} L_{n}^{0}\left(s_{1}\right) r_{2}^{m} L_{m}^{0}\left(s_{2}\right)+\text { C.C. }
\end{aligned}
$$

where

$$
\begin{gathered}
\alpha\left(k_{\|}\right)=k_{\|}^{2}-m_{f}^{2}, \\
\beta\left(k_{\|}\right)=\left(k_{\|}-p_{\|}\right)^{2}-m_{f}^{2}, \\
r_{i}=-e^{-2\left|q_{f} B\right| x_{i}}, \quad i=1,2, \\
s_{1}=\frac{2 k_{\perp}^{2}}{\left|q_{f} B\right|},
\end{gathered}
$$

By using the generating function of the Laguerre polynomials, given by

$$
s_{2}=\frac{2(k-p)_{\perp}^{2}}{\left|q_{f} B\right|} .
$$

and

$$
\sum_{n=0}^{\infty} r^{n} L_{n}^{b}(s)=\frac{1}{(1-r)^{b+1}} \exp \left(-\frac{r}{1-r} s\right)
$$

we find

$$
\begin{aligned}
t_{1}^{\mu \nu}= & -g^{2} \int \frac{d^{2} x}{\left(1+e^{-2\left|q_{f} B\right| x_{1}}\right)\left(1+e^{\left.-2\left|q_{f} B\right| x_{2}\right)}\right.} \int \frac{d^{4} k}{(2 \pi)^{4}} \exp \left[-\frac{k_{\perp}^{2}+(k-p)_{\perp}^{2}}{\left|q_{f} B\right|}\right] e^{\alpha\left(k_{\|}\right) x_{1}+\beta\left(k_{\|}\right) x_{2}} \\
& \times \exp \left[n\left(x_{1}\right) \frac{2 k_{\perp}^{2}}{\left|q_{f} B\right|}\right] \exp \left[n\left(x_{2}\right) \frac{2(k-p)_{\perp}^{2}}{\left|q_{f} B\right|}\right] \operatorname{Tr}\left\{\gamma^{\nu}\left(\not \not l_{\|}+m_{f}\right) \mathcal{O}^{-} \gamma^{\mu}\left(\not \not_{\|}-\not \not_{\|}+m_{f}\right) \mathcal{O}^{-}\right\}+\text {C.C. }
\end{aligned}
$$

where we have defined

$$
n(x) \equiv \frac{1}{e^{2\left|q_{f} B\right| x}+1} .
$$

Now, for the trace computation, note that

$$
\begin{gathered}
{\left[\gamma_{\|}^{\mu}, \mathcal{O}^{( \pm)}\right]=0,} \\
\mathcal{O}^{( \pm)} \gamma^{\mu} \mathcal{O}^{( \pm)}=\mathcal{O}^{( \pm)} \gamma_{\|}^{\mu},
\end{gathered}
$$

and therefore

$$
\begin{aligned}
& 4 \operatorname{Tr}\left\{\gamma^{\nu}\left(\not k_{\|}+m_{f}\right) \gamma_{\|}^{\mu}\left(\not \not k_{\|}-\not \not_{\|}+m_{f}\right) \mathcal{O}^{-}\right\}+\text {C.C. } \\
& \quad=4 \operatorname{Tr}\left\{\gamma^{\nu}\left(\not k_{\|}+m_{f}\right) \gamma_{\|}^{\mu}\left(\not \not_{\|}-\not \not_{\|}+m_{f}\right)\right\} \\
& \quad=16\left[\left(k_{\|} \cdot p_{\|}+m_{f}^{2}-k_{\|}^{2}\right) g_{\|}^{\mu \nu}+2 k_{\|}^{\mu} k_{\|}^{\nu}-k_{\|}^{\mu} p_{\|}^{\nu}-k_{\|}^{\nu} p_{\|}^{\mu}\right] .
\end{aligned}
$$

Putting all together

$t_{1}^{\mu \nu}=-4 g^{2} \int \frac{\mathcal{I}_{1}\left(x_{1}, x_{2}\right) \mathcal{J}_{1}^{\mu \nu}\left(x_{1}, x_{2}\right)}{\left(1+e^{-2\left|q_{f} B\right| x_{1}}\right)\left(1+e^{-2\left|q_{f} B\right| x_{2}}\right)} d^{2} x$,

with 


$$
\mathcal{I}_{1}=\int \frac{d^{2} k_{\perp}}{(2 \pi)^{2}} \exp \left[-\frac{k_{\perp}^{2}+(k-p)_{\perp}^{2}}{\left|q_{f} B\right|}\right] \exp \left[n\left(x_{1}\right) \frac{2 k_{\perp}^{2}}{\left|q_{f} B\right|}\right] \exp \left[n\left(x_{2}\right) \frac{2(k-p)_{\perp}^{2}}{\left|q_{f} B\right|}\right]
$$

and

$$
\mathcal{J}_{1}^{\mu \nu}=\int \frac{d^{2} k_{\|}}{(2 \pi)^{2}} e^{\alpha\left(k_{\|}\right) x_{1}} e^{\beta\left(k_{\|}\right) x_{2}}\left[\left(k_{\|} \cdot p_{\|}+m_{f}^{2}-k_{\|}^{2}\right) g_{\|}^{\mu \nu}+2 k_{\|}^{\mu} k_{\|}^{\nu}-k_{\|}^{\mu} p_{\|}^{\nu}-k_{\|}^{\nu} p_{\|}^{\mu}\right] .
$$

The transverse integral $\mathcal{I}_{1}$ is performed by making the shift

$$
k_{\perp}=q_{\perp}+\frac{1-2 n\left(x_{2}\right)}{2\left[1-n\left(x_{1}\right)-n\left(x_{2}\right)\right]} p_{\perp},
$$

which turns the integral into a simple Gaussian form. It is straightforward to prove that

$$
\mathcal{I}_{1}=\frac{\pi}{(2 \pi)^{2}} \frac{\left|q_{f} B\right|}{\tanh \left(\left|q_{f} B\right| x_{1}\right)+\tanh \left(\left|q_{f} B\right| x_{2}\right)} \exp \left[-\frac{\tanh \left(\left|q_{f} B\right| x_{1}\right) \tanh \left(\left|q_{f} B\right| x_{2}\right)}{\tanh \left(\left|q_{f} B\right| x_{1}\right)+\tanh \left(\left|q_{f} B\right| x_{2}\right)} \frac{p_{\perp}^{2}}{\left|q_{f} B\right|}\right] .
$$

For the parallel integral $\mathcal{J}_{1}^{\mu \nu}$, the appropriate shift is

$$
l=k_{\|}-\frac{x_{2}}{x_{1}+x_{2}} p_{\|}
$$

and by performing a rotation to Euclidean space, the integral becomes of a Gaussian form in the variable $l_{E}^{2}=l_{4}^{2}+l_{3}^{2}$, and thus

$$
\mathcal{J}_{1}^{\mu \nu}=\frac{i \pi}{(2 \pi)^{2}} \exp \left[\frac{x_{1} x_{2}}{x_{1}+x_{2}} p_{\|}^{2}-m_{f}^{2}\left(x_{1}+x_{2}\right)\right]\left[\left(\frac{x_{1} x_{2}}{\left(x_{1}+x_{2}\right)^{3}} p_{\|}^{2}+\frac{m_{f}^{2}}{x_{1}+x_{2}} g_{\|}^{\mu \nu}\right)-\frac{2 x_{1} x_{2}}{\left(x_{1}+x_{2}\right)^{3}} p_{\|}^{\mu} p_{\|}^{\nu}\right] .
$$

Collecting terms

$$
\begin{aligned}
t_{1}^{\mu \nu}= & -\frac{i\left|q_{f} B\right|}{16 \pi^{2}} g^{2} \int d^{2} x \frac{e^{\left|q_{f} B\right|\left(x_{1}+x_{2}\right)}}{\sinh \left[\left|q_{f} B\right|\left(x_{1}+x_{2}\right)\right]} \exp \left[\frac{x_{1} x_{2}}{x_{1}+x_{2}} p_{\|}^{2}-m_{f}^{2}\left(x_{1}+x_{2}\right)\right] \\
& \times \exp \left[-\frac{\tanh \left(\left|q_{f} B\right| x_{1}\right) \tanh \left(\left|q_{f} B\right| x_{2}\right)}{\tanh \left(\left|q_{f} B\right| x_{1}\right)+\tanh \left(\left|q_{f} B\right| x_{2}\right)} \frac{p_{\perp}^{2}}{\left|q_{f} B\right|}\right]\left[\left(\frac{x_{1} x_{2}}{\left(x_{1}+x_{2}\right)^{3}} p_{\|}^{2}+\frac{m_{f}^{2}}{x_{1}+x_{2}}\right) g_{\|}^{\mu \nu}-\frac{2 x_{1} x_{2}}{\left(x_{1}+x_{2}\right)^{3}} p_{\|}^{\mu} p_{\|}^{\nu}\right] .
\end{aligned}
$$

Note that the term $t_{5}^{\mu \nu}$ of Eq. (A6) has the same tensor structure as $t_{1}^{\mu \nu}$. By means of the variable shifts $m^{\prime}=m-1$ and $n^{\prime}=n-1$, which produce a factor $e^{-2\left|q_{f} B\right|\left(x_{1}+x_{2}\right)}$, this gives rise at the same set of transverse and parallel integrals as for the case of $t_{1}^{\mu \nu}$. Therefore, we can write

$$
\begin{aligned}
t_{5}^{\mu \nu}= & -\frac{i\left|q_{f} B\right|}{16 \pi^{2}} g^{2} \int d^{2} x \frac{e^{-\left|q_{f} B\right|\left(x_{1}+x_{2}\right)}}{\sinh \left[\left|q_{f} B\right|\left(x_{1}+x_{2}\right)\right]} \exp \left[\frac{x_{1} x_{2}}{x_{1}+x_{2}} p_{\|}^{2}-m_{f}^{2}\left(x_{1}+x_{2}\right)\right] \\
& \times \exp \left[-\frac{\tanh \left(\left|q_{f} B\right| x_{1}\right) \tanh \left(\left|q_{f} B\right| x_{2}\right)}{\tanh \left(\left|q_{f} B\right| x_{1}\right)+\tanh \left(\left|q_{f} B\right| x_{2}\right)} \frac{p_{\perp}^{2}}{\left|q_{f} B\right|}\right]\left[\left(\frac{x_{1} x_{2}}{\left(x_{1}+x_{2}\right)^{3}} p_{\|}^{2}+\frac{m_{f}^{2}}{x_{1}+x_{2}}\right) g_{\|}^{\mu \nu}-\frac{2 x_{1} x_{2}}{\left(x_{1}+x_{2}\right)^{3}} p_{\|}^{\mu} p_{\|}^{\nu}\right] .
\end{aligned}
$$

Adding up these two terms, we get

$$
\begin{aligned}
t_{1}^{\mu \nu}+t_{5}^{\mu \nu}= & -\frac{i\left|q_{f} B\right|}{8 \pi^{2}} g^{2} \int d^{2} x \operatorname{coth}\left[\left|q_{f} B\right|\left(x_{1}+x_{2}\right)\right] \exp \left[\frac{x_{1} x_{2}}{x_{1}+x_{2}} p_{\|}^{2}-m_{f}^{2}\left(x_{1}+x_{2}\right)\right] \\
& \times \exp \left[-\frac{\tanh \left(\left|q_{f} B\right| x_{1}\right) \tanh \left(\left|q_{f} B\right| x_{2}\right)}{\tanh \left(\left|q_{f} B\right| x_{1}\right)+\tanh \left(\left|q_{f} B\right| x_{2}\right)} \frac{p_{\perp}^{2}}{\left|q_{f} B\right|}\right]\left[\left(\frac{x_{1} x_{2}}{\left(x_{1}+x_{2}\right)^{3}} p_{\|}^{2}+\frac{m_{f}^{2}}{x_{1}+x_{2}}\right) g_{\|}^{\mu \nu}-\frac{2 x_{1} x_{2}}{\left(x_{1}+x_{2}\right)^{3}} p_{\|}^{\mu} p_{\|}^{\nu}\right] . \\
\equiv & -\frac{i}{8 \pi^{2}} g^{2} \int d^{2} x f_{0}\left(x_{1}, x_{2}\right) f_{1}^{\mu \nu}\left(x_{1}, x_{2}\right) .
\end{aligned}
$$


For the term $t_{2}^{\mu \nu}$ of Eq. (A3) the trace involved is computed by using Eq. (A17a) and the relation

$$
\mathcal{O}^{( \pm)} \gamma^{\mu} \mathcal{O}^{(\mp)}=\mathcal{O}^{( \pm)} \gamma_{\perp}^{\mu}
$$

so that

$$
\operatorname{Tr}\left\{\gamma_{\perp}^{\nu}\left(\not k_{\|}+m_{f}\right) \gamma^{\mu}\left(\not \not_{\|}-\not \not_{\|}+m_{f}\right)\right\}+\text { C.C. }=4\left(k_{\|} \cdot p_{\|}-k_{\|}^{2}+m_{f}^{2}\right) g_{\perp}^{\mu \nu} .
$$

These results imply that after introducing the Schwinger parametrization, the integration over the transverse momentum gives the same results as those in Eq. (A23). Moreover, in order to apply Eq. (A14) it is necessary to perform the shift $m^{\prime}=m-1$. That shift implies extracting a factor $-e^{-2\left|q_{f} B\right| x_{2}}$ from the sum, thus

$$
\begin{aligned}
t_{2}^{\mu \nu}= & -\frac{4 \pi\left|q_{f} B\right|}{(2 \pi)^{4}} g^{2} \int d^{2} x \frac{e^{-2\left|q_{f} B\right| x_{2}}}{\left(1+e^{-2\left|q_{f} B\right| x_{1}}\right)\left(1+e^{-2\left|q_{f} B\right| x_{2}}\right)} \frac{\left|q_{f} B\right|}{\tanh \left(\left|q_{f} B\right| x_{1}\right)+\tanh \left(\left|q_{f} B\right| x_{2}\right)} \\
& \times \exp \left[-\frac{\tanh \left(\left|q_{f} B\right| x_{1}\right) \tanh \left(\left|q_{f} B\right| x_{2}\right)}{\tanh \left(\left|q_{f} B\right| x_{1}\right)+\tanh \left(\left|q_{f} B\right| x_{2}\right)} \frac{p_{\perp}^{2}}{\left|q_{f} B\right|}\right] \int d^{2} k_{\|}\left(k_{\|} \cdot p_{\|}-k_{\|}^{2}+m_{f}^{2}\right) e^{\alpha\left(k_{\|}\right) x_{1}} e^{\beta\left(k_{\|}\right) x_{2}} g_{\perp}^{\mu \nu} .
\end{aligned}
$$

The parallel integration is carried out with the help of the momentum shift of Eq. (A24) which in Euclidean space gives

$$
\begin{aligned}
t_{2}^{\mu \nu}= & -\frac{4 i \pi^{2}\left|q_{f} B\right|}{(2 \pi)^{2}} g^{2} \int d^{2} x \frac{e^{-2\left|q_{f} B\right| x_{2}}}{\left(1+e^{-2\left|q_{f} B\right| x_{1}}\right)\left(1+e^{\left.-2\left|q_{f} B\right| x_{2}\right)} \frac{\left|q_{f} B\right|}{\tanh \left(\left|q_{f} B\right| x_{1}\right)+\tanh \left(\left|q_{f} B\right| x_{2}\right)}\right.} \\
& \times \exp \left[\frac{x_{1} x_{2}}{x_{1}+x_{2}} p_{\|}^{2}-m_{f}^{2}\left(x_{1}+x_{2}\right)\right] \exp \left[-\frac{\tanh \left(\left|q_{f} B\right| x_{1}\right) \tanh \left(\left|q_{f} B\right| x_{2}\right)}{\tanh \left(\left|q_{f} B\right| x_{1}\right)+\tanh \left(\left|q_{f} B\right| x_{2}\right)} \frac{p_{\perp}^{2}}{\left|q_{f} B\right|}\right] \\
& \times\left[\frac{x_{1} x_{2}}{\left(x_{1}+x_{2}\right)^{3}} p_{\|}^{2}+\frac{m_{f}^{2}}{x_{1}+x_{2}}+\frac{1}{\left(x_{1}+x_{2}\right)^{2}}\right] g_{\perp}^{\mu \nu} .
\end{aligned}
$$

From the fact that the term $t_{4}^{\mu \nu}$ has the same tensor structure of $t_{1}^{\mu \nu}$, it is easy to show that both expressions are related to each other after the exchange $x_{1} \leftrightarrow x_{2}$, so that

$$
\begin{aligned}
t_{4}^{\mu \nu}= & -\frac{4 i \pi^{2}\left|q_{f} B\right|}{(2 \pi)^{2}} g^{2} \int d^{2} x \frac{e^{-2\left|q_{f} B\right| x_{1}}}{\left(1+e^{-2\left|q_{f} B\right| x_{1}}\right)\left(1+e^{-2\left|q_{f} B\right| x_{2}}\right)} \exp \left[\frac{x_{1} x_{2}}{x_{1}+x_{2}} p_{\|}^{2}-m_{f}^{2}\left(x_{1}+x_{2}\right)\right] \\
& \times \exp \left[-\frac{\tanh \left(\left|q_{f} B\right| x_{1}\right) \tanh \left(\left|q_{f} B\right| x_{2}\right)}{\tanh \left(\left|q_{f} B\right| x_{1}\right)+\tanh \left(\left|q_{f} B\right| x_{2}\right)} \frac{p_{\perp}^{2}}{\left|q_{f} B\right|}\right]\left[\frac{x_{1} x_{2}}{\left(x_{1}+x_{2}\right)^{3}} p_{\|}^{2}+\frac{m_{f}^{2}}{x_{1}+x_{2}}+\frac{1}{\left(x_{1}+x_{2}\right)^{2}}\right] g_{\perp}^{\mu \nu},
\end{aligned}
$$

and therefore, after manipulating the exponential, we get

$$
\begin{aligned}
t_{2}^{\mu \nu}+t_{4}^{\mu \nu}= & -\frac{i\left|q_{f} B\right|}{8 \pi^{2}} g^{2} \int d^{2} x \frac{\cosh \left[\left|q_{f} B\right|\left(x_{2}-x_{1}\right)\right]}{\sinh \left[\left|q_{f} B\right|\left(x_{1}+x_{2}\right)\right.} \exp \left[\frac{x_{1} x_{2}}{x_{1}+x_{2}} p_{\|}^{2}-m_{f}^{2}\left(x_{1}+x_{2}\right)\right] \\
& \times \exp \left[-\frac{\tanh \left(\left|q_{f} B\right| x_{1}\right) \tanh \left(\left|q_{f} B\right| x_{2}\right)}{\tanh \left(\left|q_{f} B\right| x_{1}\right)+\tanh \left(\left|q_{f} B\right| x_{2}\right)} \frac{p_{\perp}^{2}}{\left|q_{f} B\right|}\right]\left[\frac{x_{1} x_{2}}{\left(x_{1}+x_{2}\right)^{3}} p_{\|}^{2}+\frac{m_{f}^{2}}{x_{1}+x_{2}}+\frac{1}{\left(x_{1}+x_{2}\right)^{2}}\right] g_{\perp}^{\mu \nu} \\
\equiv & -\frac{i}{8 \pi^{2}} g^{2} \int d^{2} x f_{0}\left(x_{1}, x_{2}\right) f_{2}^{\mu \nu}\left(x_{1}, x_{2}\right) .
\end{aligned}
$$

For the term $t_{3}^{\mu \nu}$, the trace is computed with the help of Eqs. (A17a), (A17b) and (A29)

$$
\operatorname{Tr}\left\{\gamma^{\nu}\left(\not k_{\|}+m_{f}\right) \mathcal{O}^{-} \gamma^{\mu}\left(\not k_{\perp}-\not p_{\perp}\right)\right\}+\text { C.C. }=4\left[k_{\|}^{\mu}\left(k_{\perp}^{\nu}-p_{\perp}^{\nu}\right)+k_{\|}^{\nu}\left(k_{\perp}^{\mu}-p_{\perp}^{\mu}\right)\right] .
$$

After introducing Schwinger's parametrization and using the generating function for the Laguerre polynomials (with the shift $m^{\prime}=m-1$ ), we obtain 


$$
\begin{aligned}
t_{3}^{\mu \nu}= & -\frac{8}{(2 \pi)^{4}} g^{2} \int d^{2} x \int d^{4} k \frac{e^{-2\left|q_{f} B\right| x_{2}} e^{\alpha\left(k_{\|}\right) x_{1}} e^{\beta\left(k_{\|}\right) x_{2}}}{\left(1+2 e^{-2\left|q_{f} B\right| x_{1}}\right)\left(1+2 e^{-2\left|q_{f} B\right| x_{1}}\right)^{2}} \exp \left[-\frac{k_{\perp}^{2}+(k-p)_{\perp}^{2}}{\left|q_{f} B\right|}\right] \\
& \times \exp \left[n\left(x_{1}\right) \frac{2 k_{\perp}^{2}}{\left|q_{f} B\right|}\right] \exp \left[n\left(x_{2}\right) \frac{2(k-p)_{\perp}^{2}}{\left|q_{f} B\right|}\right]\left[k_{\|}^{\mu}\left(k_{\perp}^{\nu}-p_{\perp}^{\nu}\right)+k_{\|}^{\nu}\left(k_{\perp}^{\mu}-p_{\perp}^{\mu}\right)\right] .
\end{aligned}
$$

The change of variable in Eq. (A22) leads to the result

$$
t_{3}^{\mu \nu}=-\frac{8}{(2 \pi)^{4}} g^{2} \int d^{2} x \int d^{4} k \frac{e^{-2\left|q_{f} B\right| x_{2}}\left[\mathcal{I}_{2}^{\mu \nu}\left(x_{1}, x_{2}\right)+\mathcal{I}_{2}^{\nu \mu}\left(x_{1}, x_{2}\right)\right]}{\left(1+2 e^{-2\left|q_{f} B\right| x_{1}}\right)\left(1+2 e^{-2\left|q_{f} B\right| x_{1}}\right)^{2}} \exp \left[-\frac{\tanh \left(\left|q_{f} B\right| x_{1}\right) \tanh \left(\left|q_{f} B\right| x_{2}\right)}{\tanh \left(\left|q_{f} B\right| x_{1}\right)+\tanh \left(\left|q_{f} B\right| x_{2}\right)} \frac{p_{\perp}^{2}}{\left|q_{f} B\right|}\right],
$$

where

$$
\mathcal{I}_{2}^{\mu \nu}=\int d^{2} k_{\|} e^{\alpha\left(k_{\|}\right) x_{1}} e^{\beta\left(k_{\|}\right) x_{2}} \int d^{2} q_{\perp} e^{-\eta q_{\perp}^{2}} k_{\|}^{\mu}\left[q_{\perp}^{\nu}+(\sigma-1) p_{\perp}^{\nu}\right]
$$

with

$$
\eta \equiv \frac{\tanh \left(\left|q_{f} B\right| x_{1}\right)+\tanh \left(\left|q_{f} B\right| x_{2}\right)}{\left|q_{f} B\right|}
$$

and

$$
\sigma \equiv \frac{\tanh \left(\left|q_{f} B\right| x_{2}\right)}{\tanh \left(\left|q_{f} B\right| x_{1}\right)+\tanh \left(\left|q_{f} B\right| x_{2}\right)} .
$$

The perpendicular integration has a simple Gaussian form for which the linear term in $q_{\perp}$ integrates to zero, yielding

$$
\mathcal{I}_{2}^{\mu \nu}=\frac{\pi(\sigma-1)}{\eta} p_{\perp}^{\nu} \int d^{2} k_{\|} e^{\alpha\left(k_{\|}\right) x_{1}} e^{\beta\left(k_{\|}\right) x_{2}} k_{\|}^{\mu}
$$

The shift of variable in Eq. (A24) also implies a Gaussian integration (in Euclidean space), where the linear terms in $l$ vanish after integration. In this way

$$
\mathcal{I}_{2}^{\mu \nu}=\frac{\pi^{2}(\sigma-1)}{\eta} \frac{x_{2}}{\left(x_{1}+x_{2}\right)^{2}} p_{\|}^{\mu} p_{\perp}^{\nu} \exp \left[\frac{x_{1} x_{2}}{x_{1}+x_{2}} p_{\|}^{2}-m_{f}^{2}\left(x_{1}+x_{2}\right)\right]=-\frac{i \pi^{2}\left|q_{f} B\right| x_{2}}{\left(x_{1}+x_{2}\right)^{2}} \frac{\tanh \left(\left|q_{f} B\right| x_{1}\right) p_{\|}^{\mu} p_{\perp}^{\nu}}{\left.\tanh \left(\left|q_{f} B\right| x_{1}\right)+\tanh \left(\left|q_{f} B\right| x_{2}\right)\right]^{2}} .
$$

Putting together these results

$$
\begin{aligned}
t_{3}^{\mu \nu}= & -\frac{i\left|q_{f} B\right| \pi^{2}}{(2 \pi)^{4}} g^{2} \int d^{2} x \frac{x_{2} e^{\left|q_{f} B\right| x_{1}} \sinh \left(\left|q_{f} B\right| x_{1}\right)}{\left(x_{1}+x_{2}\right)^{2} \sinh ^{2}\left[\left|q_{f} B\right|\left(x_{1}+x_{2}\right)\right]} \exp \left[\frac{x_{1} x_{2}}{x_{1}+x_{2}} p_{\|}^{2}-m_{f}^{2}\left(x_{1}+x_{2}\right)\right] \\
& \times \exp \left[-\frac{\tanh \left(\left|q_{f} B\right| x_{1}\right) \tanh \left(\left|q_{f} B\right| x_{2}\right)}{\tanh \left(\left|q_{f} B\right| x_{1}\right)+\tanh \left(\left|q_{f} B\right| x_{2}\right)} \frac{p_{\perp}^{2}}{\left|q_{f} B\right|}\right]\left(p_{\|}^{\mu} p_{\perp}^{\nu}+p_{\|}^{\nu} p_{\perp}^{\mu}\right) .
\end{aligned}
$$

The structure $t_{6}^{\mu \nu}$ is obtained from $t_{3}^{\mu \nu}$ after the shift $n^{\prime}=n-1$ which means introducing a factor $-e^{-2\left|q_{f} B\right| x_{1}}$, thus

$$
\begin{aligned}
t_{6}^{\mu \nu}= & -\frac{i\left|q_{f} B\right| \pi^{2}}{(2 \pi)^{4}} g^{2} \int d^{2} x \frac{x_{2} e^{-\left|q_{f} B\right| x_{1}} \sinh \left(\left|q_{f} B\right| x_{1}\right)}{\left(x_{1}+x_{2}\right)^{2} \sinh ^{2}\left[\left|q_{f} B\right|\left(x_{1}+x_{2}\right)\right]} \exp \left[\frac{x_{1} x_{2}}{x_{1}+x_{2}} p_{\|}^{2}-m_{f}^{2}\left(x_{1}+x_{2}\right)\right] \\
& \times \exp \left[-\frac{\tanh \left(\left|q_{f} B\right| x_{1}\right) \tanh \left(\left|q_{f} B\right| x_{2}\right)}{\tanh \left(\left|q_{f} B\right| x_{1}\right)+\tanh \left(\left|q_{f} B\right| x_{2}\right)} \frac{p_{\perp}^{2}}{\left|q_{f} B\right|}\right]\left(p_{\|}^{\mu} p_{\perp}^{\nu}+p_{\|}^{\nu} p_{\perp}^{\mu}\right),
\end{aligned}
$$

and therefore

$$
\begin{aligned}
t_{3}^{\mu \nu}+t_{6}^{\mu \nu}= & -\frac{i\left|q_{f} B\right|}{8 \pi^{2}} g^{2} \int d^{2} x \frac{x_{2} \cosh \left(\left|q_{f} B\right| x_{1}\right) \sinh \left(\left|q_{f} B\right| x_{1}\right)}{\left(x_{1}+x_{2}\right)^{2} \sinh ^{2}\left[\left|q_{f} B\right|\left(x_{1}+x_{2}\right)\right]} \exp \left[\frac{x_{1} x_{2}}{x_{1}+x_{2}} p_{\|}^{2}-m_{f}^{2}\left(x_{1}+x_{2}\right)\right] \\
& \times \exp \left[-\frac{\tanh \left(\left|q_{f} B\right| x_{1}\right) \tanh \left(\left|q_{f} B\right| x_{2}\right)}{\tanh \left(\left|q_{f} B\right| x_{1}\right)+\tanh \left(\left|q_{f} B\right| x_{2}\right)} \frac{p_{\perp}^{2}}{\left|q_{f} B\right|}\right]\left(p_{\|}^{\mu} p_{\perp}^{\nu}+p_{\|}^{\nu} p_{\perp}^{\mu}\right) .
\end{aligned}
$$


Coming now to the terms $t_{7}^{\mu \nu}$ and $t_{8}^{\mu \nu}$, we notice that they share a common tensor form. Starting from $t_{3}^{\mu \nu}$, the expression for $t_{7}^{\mu \nu}$ is obtained by replacing $x_{1} \rightarrow x_{2}$ and $p \rightarrow-p$. Moreover, $t_{8}^{\mu \nu}$ is obtained from $t_{7}^{\mu \nu}$ by performing the shift $m^{\prime}=m-1$ which amounts to introducing a factor $-e^{-2\left|q_{f} B\right| x_{2}}$. Implementing these observations, we get

$$
\begin{aligned}
t_{7}^{\mu \nu}+t_{8}^{\mu \nu}= & -\frac{i\left|q_{f} B\right|}{8 \pi^{2}} g^{2} \int d^{2} x \frac{x_{1} \cosh \left(\left|q_{f} B\right| x_{2}\right) \sinh \left(\left|q_{f} B\right| x_{2}\right)}{\left(x_{1}+x_{2}\right)^{2} \sinh ^{2}\left[\left|q_{f} B\right|\left(x_{1}+x_{2}\right)\right]} \exp \left[\frac{x_{1} x_{2}}{x_{1}+x_{2}} p_{\|}^{2}-m_{f}^{2}\left(x_{1}+x_{2}\right)\right] \\
& \times \exp \left[-\frac{\tanh \left(\left|q_{f} B\right| x_{1}\right) \tanh \left(\left|q_{f} B\right| x_{2}\right)}{\tanh \left(\left|q_{f} B\right| x_{1}\right)+\tanh \left(\left|q_{f} B\right| x_{2}\right)} \frac{p_{\perp}^{2}}{\left|q_{f} B\right|}\right]\left(p_{\|}^{\mu} p_{\perp}^{\nu}+p_{\|}^{\nu} p_{\perp}^{\mu}\right),
\end{aligned}
$$

then

$$
\begin{aligned}
t_{3}^{\mu \nu}+t_{6}^{\mu \nu}+t_{7}^{\mu \nu}+t_{8}^{\mu \nu} & =-\frac{i\left|q_{f} B\right|}{8 \pi^{2}} g^{2} \int \frac{d^{2} x}{2\left(x_{1}+x_{2}\right)^{2} \sinh ^{2}\left[\left|q_{f} B\right|\left(x_{1}+x_{2}\right)\right]} \exp \left[\frac{x_{1} x_{2}}{x_{1}+x_{2}} p_{\|}^{2}-m_{f}^{2}\left(x_{1}+x_{2}\right)\right] \\
& \times \exp \left[-\frac{\tanh \left(\left|q_{f} B\right| x_{1}\right) \tanh \left(\left|q_{f} B\right| x_{2}\right)}{\tanh \left(\left|q_{f} B\right| x_{1}\right)+\tanh \left(\left|q_{f} B\right| x_{2}\right)} \frac{p_{\perp}^{2}}{\left|q_{f} B\right|}\right]\left[x_{1} \sinh \left(2\left|q_{f} B\right| x_{2}\right)+x_{2} \sinh \left(2\left|q_{f} B\right| x_{1}\right)\right]\left(p_{\|}^{\mu} p_{\perp}^{\nu}+p_{\|}^{\nu} p_{\perp}^{\mu}\right) \\
& \equiv-\frac{i}{8 \pi^{2}} g^{2} \int d^{2} x f_{0}\left(x_{1}, x_{2}\right) f_{3}^{\mu \nu}\left(x_{1}, x_{2}\right)
\end{aligned}
$$

Finally, the trace in the term $t_{9}^{\mu \nu}$ is given by

$$
\operatorname{Tr}\left\{\gamma^{\nu} k_{\perp} \gamma^{\mu}\left(\not k_{\perp}-\not p_{\perp}\right)\right\}=4\left[\left(k_{\perp} \cdot p_{\perp}+k_{\perp}^{2}\right) g^{\mu \nu}+2 k_{\perp}^{\mu} k_{\perp}^{\nu}-\left(p_{\perp}^{\mu} k_{\perp}^{\nu}+p_{\perp}^{\nu} k_{\perp}^{\mu}\right)\right]
$$

After introducing the Schwinger parametrization and performing the sum together with the shift in Eq. (A22), we get

$$
\begin{aligned}
t_{9}^{\mu \nu}= & -\frac{2}{(2 \pi)^{4}} g^{2} \int \frac{d^{2} x}{\cosh ^{2}\left(\left|q_{f} B\right| x_{1}\right) \cosh ^{2}\left(\left|q_{f} B\right| x_{2}\right)} \exp \left[-\frac{\tanh \left(\left|q_{f} B\right| x_{1}\right) \tanh \left(\left|q_{f} B\right| x_{2}\right)}{\tanh \left(\left|q_{f} B\right| x_{1}\right)+\tanh \left(\left|q_{f} B\right| x_{2}\right)} \frac{p_{\perp}^{2}}{\left|q_{f} B\right|}\right] \\
& \times \int d^{2} k_{\|} e^{\alpha\left(k_{\|}\right) x_{1}} e^{\beta\left(k_{\|}\right) x_{2}} \int d^{2} q_{\perp} e^{-\eta q_{\perp}^{2}}\left[\left(q_{\perp}^{2}+\sigma(\sigma-1) p_{\perp}^{2}\right) g^{\mu \nu}+2 q_{\perp}^{\mu} q_{\perp}^{\nu}+2 \sigma(\sigma-1) p_{\perp}^{\nu} p_{\perp}^{\mu}\right],
\end{aligned}
$$

where we have ignored linear terms in $q_{\perp}$ and the variables $\eta$ and $\sigma$ are defined in Eqs. (A39). In Euclidean space, by means of the change of variable given in Eq. (A24), the parallel integral is easily performed, yielding

$$
\begin{aligned}
t_{9}^{\mu \nu}= & -\frac{2 i \pi}{(2 \pi)^{4}} g^{2} \int \frac{d^{2} x}{\left(x_{1}+x_{2}\right) \cosh ^{2}\left(\left|q_{f} B\right| x_{1}\right) \cosh ^{2}\left(\left|q_{f} B\right| x_{2}\right)} \exp \left[\frac{x_{1} x_{2}}{x_{1}+x_{2}} p_{\|}^{2}-m_{f}^{2}\left(x_{1}+x_{2}\right)\right] \\
& \times \exp \left[-\frac{\tanh \left(\left|q_{f} B\right| x_{1}\right) \tanh \left(\left|q_{f} B\right| x_{2}\right)}{\tanh \left(\left|q_{f} B\right| x_{1}\right)+\tanh \left(\left|q_{f} B\right| x_{2}\right)} \frac{p_{\perp}^{2}}{\left|q_{f} B\right|}\right] \mathcal{J}_{2}^{\mu \nu}\left(x_{1}, x_{2}\right),
\end{aligned}
$$

where

$$
\mathcal{J}_{2}^{\mu \nu}=\int d^{2} q_{\perp} e^{-\eta q_{\perp}^{2}}\left[\left(q_{\perp}^{2}+\sigma(\sigma-1) p_{\perp}^{2}\right) g^{\mu \nu}+2 q_{\perp}^{\mu} q_{\perp}^{\nu}+2 \sigma(\sigma-1) p_{\perp}^{\nu} p_{\perp}^{\mu}\right]
$$

The last integral has a simple Gaussian form and it is straightforward to compute it, yielding

$$
\begin{aligned}
\mathcal{J}_{2}^{\mu \nu}= & \frac{\pi\left|q_{f} B\right|^{2}}{\left[\tanh \left(\left|q_{f} B\right| x_{1}\right)+\tanh \left(\left|q_{f} B\right| x_{2}\right)\right]^{2}}\left[\left(1-\frac{\tanh \left(\left|q_{f} B\right| x_{1}\right) \tanh \left(\left|q_{f} B\right| x_{2}\right) p_{\perp}^{2}}{\left|q_{f} B\right|\left[\tanh \left(\left|q_{f} B\right| x_{1}\right)+\tanh \left(\left|q_{f} B\right| x_{2}\right)\right]}\right) g^{\mu \nu}\right. \\
& \left.-g_{\perp}^{\mu \nu}-\frac{2 \tanh \left(\left|q_{f} B\right| x_{1}\right) \tanh \left(\left|q_{f} B\right| x_{2}\right)}{\left|q_{f} B\right|\left[\tanh \left(\left|q_{f} B\right| x_{1}\right)+\tanh \left(\left|q_{f} B\right| x_{2}\right)\right]} p_{\perp}^{\nu} p_{\perp}^{\mu}\right]
\end{aligned}
$$


Putting all of this together, we get

$$
\begin{aligned}
t_{9}^{\mu \nu}= & -\frac{i\left|q_{f} B\right|^{2}}{8 \pi^{2}} \int \frac{d^{2} x}{\left(x_{1}+x_{2}\right) \sinh ^{2}\left[e B\left(x_{1}+x_{2}\right)\right]}\left[\left(1-\frac{\tanh \left(\left|q_{f} B\right| x_{1}\right) \tanh \left(\left|q_{f} B\right| x_{2}\right) p_{\perp}^{2}}{\left|q_{f} B\right|\left[\tanh \left(\left|q_{f} B\right| x_{1}\right)+\tanh \left(\left|q_{f} B\right| x_{2}\right)\right]}\right) g^{\mu \nu}\right. \\
& \left.-g_{\perp}^{\mu \nu}-\frac{2 \tanh \left(\left|q_{f} B\right| x_{1}\right) \tanh \left(\left|q_{f} B\right| x_{2}\right)}{\left|q_{f} B\right|\left[\tanh \left(\left|q_{f} B\right| x_{1}\right)+\tanh \left(\left|q_{f} B\right| x_{2}\right)\right]} p_{\perp}^{\nu} p_{\perp}^{\mu}\right] \\
\equiv & -\frac{i}{8 \pi^{2}} g^{2} \int d^{2} x f_{0}\left(x_{1}, x_{2}\right) f_{4}^{\mu \nu}\left(x_{1}, x_{2}\right) .
\end{aligned}
$$

\section{APPENDIX B: TENSOR MANIPULATION OF EQS. (7)}

In order to bring to light the tensor structure of Eq. (22), the terms $f_{1}^{\mu \nu}\left(x_{1}, x_{2}\right), f_{3}^{\mu \nu}\left(x_{1}, x_{2}\right)$ and $f_{4}^{\mu \nu}\left(x_{1}, x_{2}\right)$ in Eqs. (7) have been factorized in a convenient way, so as to avoid the projection procedure which can lead to nonphysical contributions. The tensor $f_{2}^{\mu \nu}\left(x_{1}, x_{2}\right)$ remains unchanged and the manipulation is made by direct inspection.

For $f_{1}^{\mu \nu}\left(x_{1}, x_{2}\right)$ :

$$
\begin{aligned}
f_{1}^{\mu \nu}\left(x_{1}, x_{2}\right) & =\left|q_{f} B\right| \operatorname{coth}\left[\left|q_{f} B\right|\left(x_{1}+x_{2}\right)\right]\left[\left(\frac{x_{1} x_{2}}{\left(x_{1}+x_{2}\right)^{3}} p_{\|}^{2}+\frac{m_{f}^{2}}{x_{1}+x_{2}}\right) g_{\|}^{\mu \nu}-\frac{2 x_{1} x_{2}}{\left(x_{1}+x_{2}\right)^{3}} p_{\|}^{\mu} p_{\|}^{\nu}\right] \\
& =\left|q_{f} B\right| \operatorname{coth}\left[\left|q_{f} B\right|\left(x_{1}+x_{2}\right)\right]\left[\left(\frac{x_{1} x_{2}}{\left(x_{1}+x_{2}\right)^{3}} p_{\|}^{2}+\frac{m_{f}^{2}}{x_{1}+x_{2}}\right) g_{\|}^{\mu \nu}+\frac{2 x_{1} x_{2}}{\left(x_{1}+x_{2}\right)^{3}}\left(p_{\|}^{2} g_{\|}^{\mu \nu}-p_{\|}^{2} g_{\|}^{\mu \nu}-p_{\|}^{\mu} p_{\|}^{\nu}\right)\right] \\
& =\left|q_{f} B\right| \frac{\operatorname{coth}\left[\left|q_{f} B\right|\left(x_{1}+x_{2}\right)\right]}{\left(x_{1}+x_{2}\right)^{3}}\left[2 x_{1} x_{2} p_{\|}^{2} \mathcal{P}_{\|}^{\mu \nu}+\left(m_{f}^{2}\left(x_{1}+x_{2}\right)^{2}-x_{1} x_{2} p_{\|}^{2}\right) g_{\|}^{\mu \nu}\right] .
\end{aligned}
$$

For $f_{3}^{\mu \nu}\left(x_{1}, x_{2}\right)$ :

$$
f_{3}^{\mu \nu}\left(x_{1}, x_{2}\right)=\frac{\left|q_{f} B\right|}{2\left(x_{1}+x_{2}\right)^{2} \sinh ^{2}\left[\left|q_{f} B\right|\left(x_{1}+x_{2}\right)\right]}\left[x_{1} \sinh \left(2\left|q_{f} B\right| x_{2}\right)+x_{2} \sinh \left(2\left|q_{f} B\right| x_{1}\right)\right]\left(p_{\|}^{\mu} p_{\perp}^{\nu}+p_{\|}^{\nu} p_{\perp}^{\mu}\right) .
$$

Notice that

$$
p^{\mu} p^{\nu}=\left(p_{\|}^{\mu}-p_{\perp}^{\mu}\right)\left(p_{\|}^{\nu}-p_{\perp}^{\nu}\right)=p_{\|}^{\mu} p_{\|}^{\nu}+p_{\perp}^{\mu} p_{\perp}^{\nu}-\left(p_{\|}^{\mu} p_{\perp}^{\nu}+p_{\|}^{\nu} p_{\perp}^{\mu}\right)
$$

therefore,

$$
\begin{aligned}
\left(p_{\|}^{\mu} p_{\perp}^{\nu}+p_{\|}^{\nu} p_{\perp}^{\mu}\right) & =p_{\|}^{\mu} p_{\|}^{\nu}+p_{\perp}^{\mu} p_{\perp}^{\nu}-p^{\mu} p^{\nu}=p_{\|}^{\mu} p_{\|}^{\nu}+p_{\perp}^{\mu} p_{\perp}^{\nu}-p^{\mu} p^{\nu}+p^{2} g^{\mu \nu}-p^{2} g^{\mu \nu} \\
& =p^{2}\left(g^{\mu \nu}-\frac{p^{\mu} p^{\nu}}{p^{2}}\right)+p_{\|}^{\mu} p_{\|}^{\nu}+p_{\perp}^{\mu} p_{\perp}^{\nu}-\left(p_{\|}^{2}-p_{\perp}^{2}\right)\left(g_{\|}^{\mu \nu}+g_{\perp}^{\mu \nu}\right) \\
& =p^{2}\left(g^{\mu \nu}-\frac{p^{\mu} p^{\nu}}{p^{2}}\right)-p_{\|}^{2} \mathcal{P}_{\|}^{\mu \nu}+p_{\perp}^{2} \mathcal{P}_{\perp}^{\mu \nu}-p_{\|}^{2} g_{\perp}^{\mu \nu}+p_{\perp}^{2} g_{\|}^{\mu \nu} \\
& =p^{2}\left(g^{\mu \nu}-\frac{p^{\mu} p^{\nu}}{p^{2}}-\mathcal{P}_{\|}^{\mu \nu}-\mathcal{P}_{\perp}^{\mu \nu}\right)+p^{2} \mathcal{P}_{\|}^{\mu \nu}+p^{2} \mathcal{P}_{\perp}^{\mu \nu}-p_{\|} \mathcal{P}_{\|}^{\mu \nu}+p_{\perp}^{2} \mathcal{P}_{\perp}^{\mu \nu}-p_{\|}^{2} g_{\perp}^{\mu \nu}+p_{\perp}^{2} g_{\|}^{\mu \nu} \\
& =p^{2} \mathcal{P}_{0}^{\mu \nu}-p_{\perp}^{2} \mathcal{P}_{\|}^{\mu \nu}+p_{\|}^{2} \mathcal{P}_{\perp}^{\mu \nu}-p_{\|}^{2} g_{\perp}^{\mu \nu}+p_{\perp}^{2} g_{\|}^{\mu \nu} .
\end{aligned}
$$

Thus,

$$
f_{3}^{\mu \nu}\left(x_{1}, x_{2}\right)=\frac{\left|q_{f} B\right|\left[x_{1} \sinh \left(2\left|q_{f} B\right| x_{2}\right)+x_{2} \sinh \left(2\left|q_{f} B\right| x_{1}\right)\right]}{2\left(x_{1}+x_{2}\right)^{2} \sinh ^{2}\left[\left|q_{f} B\right|\left(x_{1}+x_{2}\right)\right]}\left(p^{2} \mathcal{P}_{0}^{\mu \nu}-p_{\perp}^{2} \mathcal{P}_{\|}^{\mu \nu}+p_{\|}^{2} \mathcal{P}_{\perp}^{\mu \nu}-p_{\|}^{2} g_{\perp}^{\mu \nu}+p_{\perp}^{2} g_{\|}^{\mu \nu}\right)
$$

Finally, for $f_{4}^{\mu \nu}\left(x_{1}, x_{2}\right)$, given that 


$$
p_{\perp}^{\mu} p_{\perp}^{\nu}=p_{\perp}^{\mu} p_{\perp}^{\nu}+p_{\perp}^{2} g_{\perp}^{\mu \nu}-p_{\perp}^{2} g_{\perp}^{\mu \nu}=p_{\perp}^{2} \mathcal{P}_{\perp}^{\mu \nu}-p_{\perp}^{2} g_{\perp}^{\mu \nu}
$$

we have

$$
\begin{aligned}
f_{4}^{\mu \nu}\left(x_{1}, x_{2}\right)= & \frac{\left|q_{f} B\right|^{2}}{\left(x_{1}+x_{2}\right) \sinh ^{2}\left[\left|q_{f} B\right|\left(x_{1}+x_{2}\right)\right]}\left[\left(1-\frac{\tanh \left(\left|q_{f} B\right| x_{1}\right) \tanh \left(\left|q_{f} B\right| x_{2}\right)}{\left|q_{f} B\right|\left[\tanh \left(\left|q_{f} B\right| x_{1}\right)+\tanh \left(\left|q_{f} B\right| x_{2}\right)\right]} p_{\perp}^{2}\right) g^{\mu \nu}\right. \\
& \left.-g_{\perp}^{\mu \nu}-\frac{2 \tanh \left(\left|q_{f} B\right| x_{1}\right) \tanh \left(\left|q_{f} B\right| x_{2}\right)}{\left|q_{f} B\right|\left[\tanh \left(\left|q_{f} B\right| x_{1}\right)+\tanh \left(\left|q_{f} B\right| x_{2}\right)\right]} p_{\perp}^{\mu} p_{\perp}^{\nu}\right] \\
= & \frac{\left|q_{f} B\right|^{2}}{\left(x_{1}+x_{2}\right) \sinh ^{2}\left[\left|q_{f} B\right|\left(x_{1}+x_{2}\right)\right]}\left[\left(1-\frac{\tanh \left(\left|q_{f} B\right| x_{1}\right) \tanh \left(\left|q_{f} B\right| x_{2}\right)}{\left|q_{f} B\right|\left[\tanh \left(\left|q_{f} B\right| x_{1}\right)+\tanh \left(\left|q_{f} B\right| x_{2}\right)\right]} p_{\perp}^{2}\right) g^{\mu \nu}\right. \\
& \left.-g_{\perp}^{\mu \nu}-\frac{2 \tanh \left(\left|q_{f} B\right| x_{1}\right) \tanh \left(\left|q_{f} B\right| x_{2}\right)}{\left|q_{f} B\right|\left[\tanh \left(\left|q_{f} B\right| x_{1}\right)+\tanh \left(\left|q_{f} B\right| x_{2}\right)\right]} \mathcal{P}_{\perp}^{\mu \nu}+\frac{2 \tanh \left(\left|q_{f} B\right| x_{1}\right) \tanh \left(\left|q_{f} B\right| x_{2}\right)}{\left|q_{f} B\right|\left[\tanh \left(\left|q_{f} B\right| x_{1}\right)+\tanh \left(\left|q_{f} B\right| x_{2}\right)\right]} p_{\perp}^{2} g_{\perp}^{\mu \nu}\right] .
\end{aligned}
$$

By collecting the common terms of the structures $\mathcal{P}_{\|}^{\mu \nu}, \mathcal{P}_{\perp}^{\mu \nu}$ and $\mathcal{P}_{0}^{\mu \nu}$, we find the coefficients of Eqs. (23)-(27).

\section{APPENDIX C: ELIMINATION OF SPURIOUS TENSORS}

In order to eliminate the spurious contributions, we follow the procedure discussed in Ref. [22]. First, let us scale the $x$ parameters, such that $x_{i} \rightarrow \lambda z_{i}$, with $\left(\lambda, z_{i}\right) \in \mathbb{R}$. Therefore, the integral that involves the coefficient $A_{1}$ is

$$
\begin{aligned}
\mathcal{I}_{A_{1}}= & \lambda^{2} \int d^{2} z \exp \left[\lambda\left(\frac{z_{1} z_{2}}{z_{1}+z_{2}} p_{\|}^{2}-m_{f}^{2}\left(z_{1}+z_{2}\right)\right)\right] \exp \left[-\frac{\tanh \left(\lambda\left|q_{f} B\right| z_{1}\right) \tanh \left(\lambda\left|q_{f} B\right| z_{2}\right)}{\tanh \left(\lambda\left|q_{f} B\right| z_{1}\right)+\tanh \left(\lambda\left|q_{f} B\right| z_{2}\right)} \frac{p_{\perp}^{2}}{\left|q_{f} B\right|}\right] \\
& \times\left\{\frac{\operatorname{coth}\left[\lambda\left|q_{f} B\right|\left(z_{1}+z_{2}\right)\right]}{\lambda\left(z_{1}+z_{2}\right)^{3}}\left(m_{f}^{2}\left(z_{1}+z_{2}\right)^{2}-z_{1} z_{2} p_{\|}^{2}\right)+\frac{z_{1} \sinh \left(2\left|q_{f} B\right| z_{2}\right)+z_{2} \sinh \left(2 \lambda\left|q_{f} B\right| z_{1}\right)}{2 \lambda\left(z_{1}+z_{2}\right)^{2} \sinh ^{2}\left[\lambda\left|q_{f} B\right|\left(z_{1}+z_{2}\right)\right]} p_{\perp}^{2}\right. \\
& \left.+\frac{\left|q_{f} B\right|}{\lambda\left(z_{1}+z_{2}\right) \sinh ^{2}\left[\lambda\left|q_{f} B\right|\left(z_{1}+z_{2}\right)\right]}\left(1-\frac{\tanh \left(\lambda\left|q_{f} B\right| z_{1}\right) \tanh \left(\lambda\left|q_{f} B\right| z_{2}\right)}{\left|q_{f} B\right|\left[\tanh \left(\lambda\left|q_{f} B\right| z_{1}\right)+\tanh \left(\lambda\left|q_{f} B\right| z_{2}\right)\right]} p_{\perp}^{2}\right)\right\},
\end{aligned}
$$

which can be written as

$$
\begin{aligned}
\mathcal{I}_{A_{1}}= & -\lambda \frac{\partial}{\partial \lambda} \int \frac{d^{2} z}{\left(z_{1}+z_{2}\right)^{2}} \operatorname{coth}\left[\lambda\left|q_{f} B\right|\left(z_{1}+z_{2}\right)\right] \exp \left[\lambda\left(\frac{z_{1} z_{2}}{z_{1}+z_{2}} p_{\|}^{2}-m_{f}^{2}\left(z_{1}+z_{2}\right)\right)\right] \\
& \times \exp \left[-\frac{\tanh \left(\lambda\left|q_{f} B\right| z_{1}\right) \tanh \left(\lambda\left|q_{f} B\right| z_{2}\right)}{\tanh \left(\lambda\left|q_{f} B\right| z_{1}\right)+\tanh \left(\lambda\left|q_{f} B\right| z_{2}\right)} \frac{p_{\perp}^{2}}{\left|q_{f} B\right|}\right] .
\end{aligned}
$$

Scaling back $\lambda z_{1} \rightarrow x_{i}$, we obtain

$\mathcal{I}_{A_{1}}=-\lambda \frac{\partial}{\partial \lambda} \int \frac{d^{2} x}{\left(x_{1}+x_{2}\right)^{2}} \operatorname{coth}\left[\left|q_{f} B\right|\left(x_{1}+x_{2}\right)\right] \exp \left[\frac{x_{1} x_{2}}{x_{1}+x_{2}} p_{\|}^{2}-m_{f}^{2}\left(x_{1}+x_{2}\right)\right] \exp \left[-\frac{\tanh \left(\left|q_{f} B\right| x_{1}\right) \tanh \left(\left|q_{f} B\right| x_{2}\right)}{\tanh \left(\left|q_{f} B\right| x_{1}\right)+\tanh \left(\left|q_{f} B\right| x_{2}\right)} \frac{p_{\perp}^{2}}{\left|q_{f} B\right|}\right]$,

and thus, the derivative is applied to a function independent of $\lambda$. Therefore $\mathcal{I}_{A_{1}}=0$.

The implementation of the same argument for $\mathcal{I}_{A_{2}}$ is more involved, given that the function is not a trivial combination of coefficients for $p_{\|}^{2}$ and $p_{\perp}^{2}$. After the $\lambda$-scaling, the integral is

$$
\mathcal{I}_{A_{2}}=\lambda^{2} \int \frac{d^{2} z}{\lambda\left(z_{1}+z_{2}\right)^{2}} I\left(\lambda z_{1}, \lambda_{2}\right)
$$

where 


$$
\begin{aligned}
I\left(\lambda z_{1}, \lambda z_{2}\right)= & f_{0}\left(\lambda z_{1}, \lambda z_{2}\right)\left[\frac{\cosh \left[\lambda\left|q_{f} B\right|\left(z_{2}-z_{1}\right)\right]}{\sinh \left[\lambda\left|q_{f} B\right|\left(z_{1}+z_{2}\right)\right]}\left(\frac{z_{1} z_{2}}{z_{1}+z_{2}} p_{\|}^{2}+m_{f}^{2}\left(z_{1}+z_{2}\right)+\frac{1}{\lambda}\right)\right. \\
& \left.-\frac{z_{1} \sinh \left(2 \lambda\left|q_{f} B\right| z_{2}\right)+z_{2} \sinh \left(2 \lambda\left|q_{f} B\right| z_{1}\right)}{2 \sinh ^{2}\left[\lambda\left|q_{f} B\right|\left(z_{1}+z_{2}\right)\right]} p_{\|}^{2}+\frac{\left(z_{1}+z_{2}\right) \sinh \left(\lambda\left|q_{f} B\right| z_{1}\right) \sinh \left(\lambda\left|q_{f} B\right| z_{2}\right)}{\sinh ^{3}\left[\lambda\left|q_{f} B\right|\left(z_{1}+z_{2}\right)\right]} p_{\perp}^{2}\right],
\end{aligned}
$$

so that by expanding in a Taylor series around $\lambda=0$ it is possible to find that

$$
\begin{aligned}
\int I\left(\lambda z_{1}, \lambda z_{2}\right) d \lambda= & -\frac{1}{\left|q_{f} B\right|\left(z_{1}+z_{2}\right) \lambda}+\frac{2\left|q_{f} B\right|^{2}\left(z_{1}+z_{2}\right)^{2}\left(z_{1}^{2}-4 z_{1} z_{2}+z_{2}^{2}\right)+3\left(z_{1} z_{2} p^{2}-m^{2}\left(z_{1}+z_{2}\right)^{2}\right)^{2}}{6\left|q_{f} B\right|\left(z_{1}+z_{2}\right)^{3}} \lambda \\
& +\frac{\lambda^{2}}{6\left|q_{f} B\right|\left(z_{1}+z_{2}\right)^{4}}\left[\left(3 p^{4} z_{1}^{2} z_{2}^{2}-3 m^{2} p^{2} z_{1} z_{2}\left(z_{1}+z_{2}\right)^{2}+m^{4}\left(z_{1}+z_{2}\right)^{4}\right)\left(z_{1}+z_{2}\right)^{2} m^{2}\right. \\
& \left.-z_{1} z_{2}\left(p^{6} z_{1}^{2} z_{2}^{2}-2\left|q_{f} B\right|^{2}\left(z_{1}+z_{2}\right)^{2}\left(p^{2}\left(z_{1}-z_{2}\right)^{2}-p_{\perp}^{2} z_{1} z_{2}\right)\right)\right] \\
& +\frac{\lambda^{3}}{1080\left|q_{f} B\right|\left(z_{1}+z_{2}\right)^{5}}\left[45\left(p^{2} z_{1} z_{2}-m^{2}\left(z_{1}+z_{2}\right)^{2}\right)^{4}+8\left|q_{f} B\right|^{4}\left(z_{1}+z_{2}\right)^{4}\left(z_{1}^{4}+4 z_{1}^{3} z_{2}-24 z_{1}^{2} z_{2}^{2}+4 z_{1} z_{2}^{3}+z_{2}^{4}\right)\right. \\
& -60\left|q_{f} B\right|^{2}\left(z_{1}+z_{2}\right)^{2}\left(p^{2} z_{1} z_{2}-m^{2}\left(z_{1}+z_{2}\right)^{2}\right)\left(m^{2}\left(z_{1}+z_{2}\right)^{2}\left(z_{1}^{2}-4 z_{1} z_{2}+z_{2}^{2}\right)\right. \\
& \left.\left.+z_{1} z_{2}\left(p^{2}\left(3 z_{1}^{2}-4 z_{1} z_{2}+3 z_{2}^{2}\right)-6 p_{\perp}^{2} z_{1} z_{2}\right)\right)\right]+\mathcal{O}\left(\lambda^{4}\right),
\end{aligned}
$$

where the desired scaling properties are recovered and hold for all orders in $\lambda$. This means that it is possible to write

$$
\int I\left(\lambda z_{1}, \lambda z_{2}\right) d \lambda=-\frac{1}{\left|q_{f} B\right|\left(z_{1}+z_{2}\right) \lambda}+h\left(\lambda z_{1}, \lambda z_{2}\right)
$$

thus

$$
I\left(\lambda z_{1}, \lambda z_{2}\right)=\frac{\partial}{\partial \lambda}\left[-\frac{1}{\left|q_{f} B\right|\left(z_{1}+z_{2}\right) \lambda}+h\left(\lambda z_{1}, \lambda z_{2}\right)\right]=\frac{\partial}{\partial \lambda}\left[-\frac{1}{\left|q_{f} B\right|\left(x_{1}+x_{2}\right)}+h\left(x_{1}, x_{2}\right)\right]=0,
$$

and therefore, $\mathcal{I}_{A_{2}}=0$.

The above argument is valid for all values of $\lambda$. Consequently, the result can be taken as general.

[1] G. S. Bali, F. Bruckmann, G. Endrődi, Z. Fodor, S. D. Katz, and A. Schäfer, Phys. Rev. D 86, 071502(R) (2012).

[2] N. O. Agasian, Phys. Lett. B 562, 257 (2003).

[3] A. O. Starinets, A. V. Vshivtsev, and V. Ch. Zukovskii, Phys. Lett. B 322, 403 (1994).

[4] V. V. Skalozub and A. V. Strelchenko, Eur. Phys. J. C 33, 105 (2004).

[5] L. McLerran and V. Skokov, Nucl. Phys. A929, 184 (2014).

[6] V. Skokov, A. Illarionov, and V. Toneev, Int. J. Mod. Phys. A 24, 5925 (2009).

[7] D. E. Kharzeev, L. D. McLerran, and H. J. Warringa, Nucl. Phys. A803, 227 (2008).

[8] A. Ayala, J. D. Castaño-Yepes, C. A. Dominguez, L. A. Hernandez, S. Hernandez-Ortiz, and M.E. TejedaYeomans, Phys. Rev. D 96, 014023 (2017); 96, 119901(E)
(2017); A. Ayala, J. D. Castaño-Yepes, C. A. Dominguez, and L. A. Hernandez, EPJ Web Conf. 141, 02007 (2017).

[9] G. Basar, D. E. Kharzeev, and V. Skokov, Phys. Rev. Lett. 109, 202303 (2012).

[10] G. Basar, D. E. Kharzeev, and E. V. Shuryak, Phys. Rev. C 90, 014905 (2014).

[11] B. G. Zakharov, Eur. Phys. J. C 76, 609 (2016).

[12] K. Tuchin, Phys. Rev. C 91, 014902 (2015).

[13] L. McLerran and B. Schenke, Nucl. Phys. A929, 71 (2014).

[14] K. Fukushima, Phys. Rev. D 83, 111501(R) (2011).

[15] A. Bandyopadhyay, C. A. Islam, and M. G. Mustafa, Phys. Rev. D 94, 114034 (2016).

[16] A. Ayala, C. A. Dominguez, S. Hernandez-Ortiz, L. A. Hernandez, M. Loewe, D. Manreza Paret, and R. Zamora, arXiv:1805.07344. 
[17] K. Hattori and K. Itakura, Ann. Phys. (Amsterdam) 330, 23 (2013).

[18] V. A. Miransky and I. A. Shovkovy, Phys. Rep. 576, 1 (2015).

[19] V. E Gusynin, V. A. Miransky, and I. A. Shovkovy, Nucl. Phys. B462, 249 (1996).

[20] E. J. Ferrer and A. Sanchez, Phys. Rev. D 100, 096006 (2019).

[21] K. Hattori and D. Satow, Phys. Rev. D 97, 014023 (2018).

[22] J. D. Bjorken and S.D. Drell, Relativistic Quantum Mechanics I (McGraw-Hill, New York, 1964).
[23] K.-I. Ishikawa, D. Kimura, K. Shigaki, and A. Tsuji, Int. J. Mod. Phys. A 28, 1350100 (2013).

[24] P. K. Jain, Y. Xiao, R. Walsworth, and A. E. Cohen, Nano Lett. 9, 1644 (2009).

[25] W. Dittrich and M. Reuter, Lecture Notes in Physics (Springer-Verlag, Berlin, 1985), Vol. 220.

[26] R. P. Mignani, V. Testa, D. González Caniulef, R. Taverna, R. Turolla, S. Zane, and K. Wu, Mon. Not. R. Astron. Soc. 465, 492 (2017). 